Ebisu Ebisu

Études japonaises Études japonaises

50 | automne-hiver 2013

Création et valeurs dans le Japon moderne

\title{
L'universalisme de l'esthétique chez Okakura Kakuzō (dit Tenshin) et Ernest Fenollosa : critique et actualité
}

岡倉覚三（「天心」）とアーネスト・F・フエノロサの美学理論の普 遍主義一現代美学の钼点力らの考察

The Quest for an Objective yet Multiple Standard of Artistic Beauty: Questioning Okakura Kakuzō (Tenshin) and Ernest F. Fenollosa

\section{Arthur Mitteau}

\section{OpenEdition}

\section{Journals}

Édition électronique

URL : http://journals.openedition.org/ebisu/1138

DOI : 10.4000/ebisu. 1138

ISSN : 2189-1893

Éditeur :

Institut français de recherche sur le Japon (UMIFRE 19 MAEE-CNRS), Maison franco-japonaise

Édition imprimée

Date de publication : 1 octobre 2013

Pagination : $95-133$

ISSN : 1340-3656

Référence électronique

Arthur Mitteau, «L'universalisme de l'esthétique chez Okakura Kakuzō (dit Tenshin) et Ernest

Fenollosa : critique et actualité », Ebisu [En ligne], 50 | automne-hiver 2013, mis en ligne le 21 juin 2014 consulté le 19 avril 2019. URL : http://journals.openedition.org/ebisu/1138 ; DOI : 10.4000/ebisu.1138 


\title{
L'universalisme de l'esthétique chez Okakura Kakuzō (dit Tenshin) et Ernest Fenollosa
}

\section{Critique et actualité}

\author{
Arthur MITTEAU*
}

Pour Tan.o Yasunori

Si l'on appelle " universalisme " la démarche qui consiste à s'appuyer en sciences humaines sur des concepts supposés universels, assortie de la croyance en la pertinence d'une telle démarche, alors il faut constater que les positions universalistes ne sont, en art, plus recevables sans passer par le crible préalable d'une importante critique. Non seulement le recours à une telle notion a longtemps fait écran à la variété des approches, dans les diverses cultures, de ce que l'on appelle " art ", mot qui recouvre un ensemble d'institutions et de pratiques dont l'unité même reste à éclairer ; non seulement cette démarche a pu servir à imposer certaines valeurs esthétiques au détriment d'autres ; mais de plus, elle tend à devenir une valeur en elle-même, dont les enjeux dépassent les questions d'art, mais reviennent cependant compliquer celles-ci. D'un autre côté, il y a bien, au Japon comme en France, des musées et des institutions patrimoniales, qui exposent des œuvres. Le choix de ces objets implique l'idée que ceuxci contiennent un intérêt par eux-mêmes, et comme il consiste à les soumettre à un public par essence infini, ce choix leur confère une valeur de fait universelle. Cette valeur n'est certes pas nécessairement esthétique, mais comme l'esthétique, au sens de l'appréciation d'une forme, peut bien être, en France comme au Japon, un mode d'appréhension des objets par lequel nous pouvons réagir par exemple lorsque nous allons dans un musée d'art,

* INALCO, Centre d'études japonaises. 
l'institution muséale confère, sauf indication contraire, au moins une légitimité au point de vue esthétique sur les œuvres qu'elle consacre. Dans le langage contemporain aussi, avec le terme " chef-d'œuvre " en français ou des mots comme kessaku 傑作 (" œuvre remarquable, saillante ") ou meisaku 名作 ( œuvre célèbre ») en japonais moderne, il existe des termes qui semblent renvoyer à une valeur artistique sinon objective, du moins transmissible à autrui.

On peut présenter l'approche esthétique comme la position consistant à définir la valeur d'une ouvre du point de vue de spectateurs n'étant pas a priori instruits des règles d'appréciation en vigueur dans le contexte de genèse de cette œuvre, par opposition à l'approche qui juge selon des critères éclairés par ce contexte, et que l'on pourrait appeler point de vue du connaisseur ou encore "poḯtique " (selon par exemple l'analyse d'Yves Michaud 2003 : 49-50). Ainsi formulé, on pressent que ce point de vue esthétique ne peut manquer d'être confronté à des problèmes si l'on cherche à l'ériger en discours théorique sur l'art, mais d'un autre côté, y renoncer serait délégitimer l'approche spontanée que l'on pourrait avoir de l'objet par ce biais. Est-il donc possible de construire une théorie esthétique viable, c'est-à-dire dont l'universalisme postulé soit compatible de fait avec la diversité des critères de l'appréciation artistique, donc un universalisme réel ?

Le présent article étudie dans une telle perspective les thèses de deux théoriciens, le professeur de philosophie, de logique et d'économie politique, puis conservateur américain Ernest F. Fenollosa (1853-1908), et son disciple, professeur d'histoire de l'art, directeur d'école d'art et conservateur, Okakura Kakuzō 岡倉覚三, dit Tenshin ${ }^{1}$ 天心 (1862-1963), lors de ce que l'on a pu appeler la « réaction nationaliste »(Charrier 1999: 163) du monde des arts dans le Japon de l'ère Meiji. Derrière cette dénomination, qu'il ne faut pas prendre de façon réductrice puisque Charrier montre elle-même que les tendances nationalistes et conservatrices du mouvement cohabitent avec des thèses syncrétiques et un souci de création d'un art nouveau, se trouvent des théoriciens, des administrateurs et des artistes qui ont tenté de penser l'art japonais ancien pour en poser la légitimité, face à ce qu'ils ont vécu comme la menace, dans la décennie 1870, de l'art occidental représenté par ses courants alors dominants : académisme et réalisme.

1. Sur ce surnom et ses implications, voir note 6 . 
Une telle approche de ces auteurs du point de vue de la philosophie esthétique est inhabituelle, dans la mesure où ils sont en général étudiés sous un angle essentiellement historique, depuis le travail pionnier de Kurihara Shin.ichi (1963), suivis de ceux de Takashina Shūji (1990 première édition 1973 ; 1993), de Murakata Akiko (1983, 2000-2001) et Yamaguchi Seiichi (1984). Il en va de même pour les principales études publiées en français sur ces auteurs, que ce soit les travaux d'Isabelle Charrier $(1991,1999)$ et ceux de Christophe Marquet (1995, 1999, 2002, 2005), ou les publications en japonais ou en anglais, qui cherchent essentiellement à mieux comprendre ces auteurs à partir de leur contexte, tout en éclairant en retour celui-ci, et l'évolution du monde des arts, à la lumière de leurs théories, y compris en jetant un regard critique sur elles. L'aspect critique de cette approche est en particulier développé par certaines études américanophones récentes (Rimer 2001, Weston 2004, Conant 2006, Croissant 2006, Guth 2006). $\mathrm{Au}$ Japon, la tendance actuelle cherche à concilier approche historique critique et tentatives de tirer un bilan, de comprendre ce que ces deux auteurs ont à apporter aujourd'hui, au Japon et peut-être au-delà, en particulier chez certains commentateurs comme Kinoshita Nagahiro et Inaga Shigemi. Puisqu'il se pose la question de l'intérêt actuel des thèses de ces auteurs, le présent article se situe dans le fil de leur démarche, malgré certains désaccords d'interprétation². S'appuyer sur les apports des études plus critiques, comme celles de l'école américaine (représentée ici par J. Thomas Rimer ou Ellen Conant), s'avère d'autant plus nécessaire ${ }^{3}$.

2. Nous pensons qu'il est hâtif de conclure que la pensée d'Okakura soit vouée à passer pour un échec en Occident parce qu'elle semblerait soit trop occidentalisée et donc hors de propos, soit irrationnelle dès qu'elle se risque à proposer un exemple de "pensée orientale typique " ("some typically Oriental thinking ", Inaga $2011: 40-41$ chapitre "The Western Empire fights back »). Penser cela présuppose (sans le prouver) que ce qui est nommé philosophie soit par essence occidental et condamné à rejeter tout ce qui serait étranger à sa "notion même " (" the very notion of philosophy»), or de Platon et Dōgen à Kant et Nishida, il nous semble que la philosophie ou les philosophies consistent bien plus à tenter de parler de ce qui les dépasse, qu’à l'exclure comme étranger.

3. Pour un aperçu des recherches récentes sur Okakura, voir les ouvrages collectifs "Okakura Tenshin. Geijutsu kyōiku no ayumi " ten jikkō iinkai (2010), Okakura Tenshin kenkyūkai (2005), en japonais, et Lippit \& Murai (2012) en anglais (qui inclut les travaux les plus récents de certains chercheurs japonais). Voir aussi Shimizu (2012). 
Après avoir montré en quoi Fenollosa a pu, avec l'aide d'Okakura, développer dans les premières décennies de l'ère Meiji une théorie esthétique qui intègre l'idée d'une pluralité des critères de valeur, l'étude de cas concrets du genre de la "calligraphie " montrera les limites de cette théorie en matière de pluralisme, et partant ses contradictions. On cherchera alors chez Okakura Tenshin des signes de tentatives de dépasser un tel problème.

\section{Un universalisme justifié, parce que fondé sur un pluralisme?}

Parler d'universalité des valeurs artistiques renvoie immédiatement à un certain nombre de questions sous-jacentes. Une première ambiguïté doit être évitée : " universalité " ne signifie pas que certaines valeurs esthétiques déterminées soient au-dessus de tous les autres par principe, et que ces valeurs devraient à ce titre s'imposer à tous. Autrement dit, il ne s'agit pas d'affirmer la supériorité exclusive, unilatérale de certaines œuvres ou genres, issus de cultures ou d'époques déterminées, sans que cette supériorité ne puisse jamais être discutée. Il s'agit plutôt de conserver un concept alternatif de l'" universalité » des valeurs artistiques qui signifie qu'une personne $\mathrm{x}$ peut apprécier la même œuvre que moi selon des critères similaires ou apparentés, et que ces critères déclenchent chez elles une appréciation qui nous sera donc, en ce sens, commune. Par exemple, nous pouvons voir l'un comme l'autre que l'allongement des figures chez Greco, couplé à la torsion de leur physionomie, et les couleurs bleues, inquiètes peut-être (car nous aurons peut-être vu l'un et l'autre l'interprétation possible, ici, du bleu comme signe de cette inquiétude indéfinissable), produit tel effet, qui nous semble bien particulier, mais, à l'un comme l'autre, appréciable.

Ainsi formulé, il apparaît qu'une telle théorie consiste en fait à définir une forme acceptable d'objectivisme, en tenant toutefois compte de la recommandation de Cassirer ${ }^{4}$ de bien définir la beauté (qui désigne ici simplement la valeur esthétique) comme un rapport entre le sujet et l'objet, qui implique l'un aussi bien que l'autre : il ne s'agit pas de tomber dans un réalisme naïf, mais de laisser, par cette définition, une part importante aux

4. Dans "Qu’est-ce que la beauté " (Cassirer 1995 : 123). 
données historiques et sociologiques, aussi bien qu'individuelles. On peut caractériser les critères "objectifs " ainsi entendus comme 1) issus de la façon dont je vois l'objet, et dont autrui pourrait également le voir ; 2) pouvant être communs, partagés avec d'autres spectateurs, même si leur transmission peut certes poser problème ; 3 ) corrélatifs au jugement conscient de l'individu, contigus avec celui-ci en tant que sujet de la connaissance. Mais un tel modèle continue à ne pas aller de soi, et demeure à bon droit sujet aux objections de type relativiste ${ }^{5}$. Pour cette raison, et à cause des interprétations culturo-centrées auquel il peut donner lieu, l'universalisme qui semble pouvoir découler logiquement d'une telle esthétique objectiviste peut paraître problématique. Pourtant, en suivant la définition ci-dessus, il en résulte que cet universalisme consiste surtout à définir un champ esthétique commun, c'est-à-dire une possibilité de droit pour certaines valeurs esthétiques d'être transmises, comprises au-delà de leur champ culturel d'origine. Non seulement cela n'exclut pas, mais cela implique une dimension de pluralité, de diversité des goûts, aussi bien que des formes et des pratiques artistiques. Mais pour respecter cela, et garantir ainsi que l'universalisme défini soit bien réel, il resterait à élaborer une théorie qui rende compte et maintienne ensemble ces deux éléments : une prise en compte de la diversité réelle des critères et des pratiques, et la possibilité de trouver certaines formes artistiques dignes de valeur pour des raisons que l'on pense universellement transmissibles.

C'est dans une telle perspective que nous nous proposons de lire les thèses esthétiques d'Okakura Kakuzō, le futur "Tenshin ", et d'Ernest Fenollosa. Il s'agit de les prendre comme un cas d'étude problématique de tentative de construction d'un tel modèle. En général ces textes ne sont pas étudiés sous cet angle, mais plutôt dans une perspective historique critique, à cause du rôle qu'ils ont joué, et continuent de jouer ${ }^{6}$, dans la construction

5. Un exemple contemporain de théorie relativiste, souvent cité dans la littérature francophone, peut être trouvé chez Genette (1997). Concernant le troisième critère, par exemple, son relativisme est inséparable de sa thèse selon laquelle les raisons de l'appréciation esthétique sont au fond exclusivement inconscientes (dans une optique freudienne : Proust aimant tel goût en raison de son enfance, etc.)

6. Kinoshita Nagahiro a déterminé que «Tenshin » était en fait le hōmyō 法名 (nom de religion, donné après la mort) d'Okakura, et fait remarquer qu'il ne fut pas, à quelques exceptions près, employé de son vivant comme nom de plume (Kinoshita 2005 : 1-3), 
d'une " essence " nationale japonaise. Une raison en est à chercher dans l'histoire du Japon moderne, certains théoriciens de l'impérialisme de l'ère Shōwa ayant directement fait référence à "Tenshin ", construisant même, si l'on en croit Kinoshita Nagahiro, entièrement le personnage auquel correspond ce nom. Mais ce qui a rendu possible cela, c'est bien la façon dont Okakura Kakuzō et Ernest Fenollosa ont délibérément pris parti dans les polémiques de leur temps, et accepté d'incarner un côté, un parti dans ces polémiques, celui de la défense d'un art national supposé « authentique ${ }^{7}$ " contre l'influence de l'académisme issu des écoles occidentales.

De cette prise de position, il résulte qu'il peut sembler de prime abord difficile de trouver chez ces auteurs une théorie esthétique " universelle ", au sens à la fois d'englobante et de pluraliste : la critique de l'art occidental académique peut y être si virulente que ce qui en résulte est l'impression d'une partialité incompatible avec tout recul théorique. C'est le cas en particulier pour le texte fondateur de ce mouvement, Bijutsu shinsetsu 美術真説 (Discours sur la vraie théorie des arts, Fenollosa 1989), qui est la traduction en japonais d'un discours prononcé par Fenollosa le 14 mai 1882 à Ueno devant une audience comprenant nombre d'officiels, dont le ministre de l'Instruction publique Fukuoka Takachika 福岡孝弟 (en fonction de 1881 à 1883). Nous n'entrerons pas ici dans les détails des arguments du discours ou du contexte de sa prononciation puis (encore plus important) de sa traduction, renvoyant à d'autres exposés ${ }^{8}$. Ce qu'il faut noter c'est que Fenollosa, si l'on en croit le texte japonais - qui n'est bien sûr pas de sa main ${ }^{9}$ - élabore dans un premier temps une théorie générale du bel art,

alors que c'est le nom que retint la postérité, y compris les nationalistes comme Yasuda Yojūrō 保田與重郎 qui voulurent faire d'Okakura un précurseur de l'impérialisme de Shōwa. Kinoshita propose par conséquent d'en bannir l'usage, considérant qu'au Japon le travail de mémoire n'est pas achevé. Ici «Tenshin » est encore ponctuellement employé, pour faire le lien avec ce personnage connu des études japonaises françaises sous ce nom, mais inconnu au-delà. Mais il est de toute façon préférable de se référer à son nom de naissance, Okakura Kakuzō, dès lors que l'on veut dégager le penseur réel et ses idées, du personnage qui a été construit par la suite.

7. C'est ainsi que nous traduisons shinsei 真誠 (dans Fenollosa 1989: 58).

8. Kurihara 1963:311-365; Taki 1993 : 110-119; ainsi que Charrier 1999: 164-167 et Rimer 2001 pour des résumés et commentaires plus critiques.

9. Le texte japonais est une traduction officiellement attribuée à Ōmori Korenaka (ou Ichū) 大森惟中, bien que l'on puisse considérer comme au moins aussi important le 
qu'il oppose aussi bien à la démonstration de virtuosité qu'à l'artisanat à visée fonctionnelle, en proposant de voir dans l'essence de ce bel art l'expression d'un "idéal ${ }^{10}$ " au moyen d'une forme (laquelle constitue, avec la perception correspondante, ce qu'il appelle une "idée ») dont le caractère parfait doit résulter à la fois de son unité organique ${ }^{11}$ et de sa capacité à exprimer correctement, par des effets plastiques, musicaux ou poétiques, un tel idéal. Puis, dans un second temps, Fenollosa s'efforce d'appliquer ces critères pour montrer que la peinture japonaise ancienne en particulier, par opposition à la peinture à l'huile d'origine occidentale, correspond à une application réussie de ces critères, en une énonciation de dix principes généraux puis de cinq points de comparaison destinés à rendre éclatante la supériorité de la première sur la seconde. Ces cinq derniers points, qui constituent peut-être le passage le plus célèbre, peuvent être résumés ainsi : en 1) ne cherchant pas la représentation exacte de la nature extérieure (on dirait mimesis) ; 2) préférant au clair-obscur une utilisation expressive du

rôle d'Okakura Kakuzō et d'Ariga Nagao 有賀長雄, alors tout jeunes étudiants attachés à servir d'interprètes, de traducteurs et d'aides auprès de leur professeur américain. Les choix de traduction qui y ont été faits étaient à la fois novateurs en terme de vocabulaire, avec parfois des partis pris qui ont pu faire dire que l'équipe japonaise infléchissait ou du moins déterminait dans un sens particulier le sens du discours (Croissant 2006 : 164), d'autant que le manuscrit anglais de la main de Fenollosa est perdu. Murakata Akiko a cependant trouvé plusieurs autres manuscrits, dont un en particulier daté de 1881, qui constitueraient de toute évidence la base du texte de la conférence de 1882 (Murakata 1983) : toutes les grandes lignes y sont, avec parfois des formules éclairantes. Quoi qu'il en soit, cet épisode montre qu'Okakura fut, dès le début, partie prenante de la construction de la pensée de Fenollosa, d'autant qu'en tant qu'interprète de celui-ci, il put à loisir l'influencer par la façon dont il présentait les informations que l'Américain recevait de ses maitres japonais en matière d'art, apprenant sans doute lui-même, à cette occasion, une bonne partie de ses connaissances dans ce domaine.

10. Ce terme "idéal " que l'on trouve fréquemment dans les textes anglais des deux auteurs, est rendu dans la version japonaise du discours de 1882 par le terme myōri 妙理, et il sera par la suite traduit par riso 理想 qui est encore en usage. Il renvoie partiellement à Hegel, et désigne en fait chez Okakura comme chez Fenollosa une idée particulière, déterminée, mais qui désigne toujours une interprétation globale du monde, de l'expérience humaine ou de la société, connotée de ce fait d'une valeur sociale, religieuse ou philosophique.

11. Au sens des philosophes de l'esthétique moderne comme Kant, chaque partie (de l'œuvre, comme d'un organisme) est nécessaire à l'existence du tout, mais le tout fait aussi vivre - et dans le cas de l'œuvre, donne son sens aux parties. 
contraste (nōtan 濃淡) ; 3) recourant à l'usage de la ligne à l'encre noire pour les contours (kōroku 鈳勒), ce qui déboucherait sur des formes moins naturelles mais mieux saisissables par l'esprit ; 4) ne recherchant pas forcément une extrême richesse de coloris ; 5) privilégiant en général la concision et l'économie de moyens sur l'exhaustivité, pour toutes ces raisons (ou du moins, ce que Fenollosa voit comme des caractéristiques essentielles), la peinture japonaise " authentique " serait en général mieux à même d'exprimer une " idée » que la peinture à l'huile d'inspiration occidentale, et donc supérieure (Fenollosa 1989 : 53-56). On a bien dans ce texte une esthétique générale, présentant une théorie unifiée de l'appréciation artistique dans laquelle le terme de beau joue un rôle central, bien que ne représentant pas le tout de l'expérience artistique; pourtant, cette théorie semble viser à la défense et à l'illustration d'une seule et unique école, de façon surtout polémique et partiale. Voilà pourquoi on a beaucoup présenté le courant de Fenollosa et de ses proches et disciples, dont "Tenshin », comme résultant essentiellement d'une application de la théorie esthétique d'origine occidentale destinée à la promotion de l'art japonais, selon une logique historico-politique qui se comprend très bien.

On peut toutefois se demander si, derrière un tel simplisme de façade, sans doute accentué par le caractère politique du texte, la vision des arts d'Ernest Fenollosa n'aurait pas été plus complexe. Lui-même aurait-il d'ailleurs cru à ses propres idées si ce n'était pas le cas ? Se serait-il vraiment intéressé aux arts, au point d'écrire à vingt-cinq ans un discours aussi élaboré que celui de 1882, s’il n’avait rien apprécié des genres artistiques occidentaux, y compris sous la forme d'un classicisme académique qui régnait alors dans sa Nouvelle-Angleterre natale ? Plus fondamentalement, ancrer la valeur artistique d'œuvres japonaises anciennes appelées à devenir des canons, les classiques de la culture du Japon moderne à venir, dans une théorie solide ne pouvait se faire en rejetant purement et simplement l'idée de toute valeur extérieure. Le procédé aurait été bien trop simpliste : pour fonder de façon absolue l'idée de la valeur de ces œuvres, il fallait les appuyer sur une théorie dont le caractère universel se manifeste aussi bien par la méthode elle-même, conceptuelle et héritée de la philosophie esthétique dans une ligne inspirée de Kant et de Hegel, que par une ouverture réelle à des œuvres issues d'horizons réellement divers, par des références seules à même de plaider pour le caractère concret de cette universalité, et ainsi pour sa véracité. Or, si l'on prend les textes de Fenollosa dans leur ensemble, qu'on les complète par la 
consultation de ses manuscrits, on peut remarquer tout une série de processus qui passent plus par la comparaison, le rapprochement de certaines œuvres occidentales et d'œuvres chinoises ou japonaises que par le rejet unilatéral des premières, selon des finalités variées, mais d'où se dégage la structure suivante : des œuvres japonaises, chinoises, coréennes d'une part, et européennes d'autre part, sont comparées soit simplement au nom d'un rang de valeur considéré comme équivalent à l'intérieur de leurs paradigmes respectifs, soit parfois de façon plus déterminée, sous l'argument d'une supposée analogie stylistique ${ }^{12}$. Sans commenter le bien-fondé de tels rapprochements, on peut noter que cette structure analogique tend à poser, en fait, quatre catégories : des œuvres orientales et occidentales de qualité, et des œuvres occidentales et orientales jugées de mauvaise qualité. Les commentateurs ont donc tôt fait de remarquer que Fenollosa, ou même Okakura après lui, cherchaient moins à rejeter en bloc l'art occidental ou même certaines tendances chez celui-ci, qu’à définir des critères qui fassent apparaittre un nouveau modèle, lequel pourrait en retour servir de référence pour l'évolution à venir des arts en Europe et en Amérique aussi bien quau Japon. C'est ainsi que Takashina Shūji remarque dès 1973 que les arguments développés dans la partie critique contre la peinture à l'huile peuvent dans une certaine mesure être présentés comme réversibles, utilisables en faveur de certaines œuvres occidentales et contre certaines peintures ou estampes japonaises (Takashina 1990 : 215), en tout cas pour ceux qui s'appuient sur l'idée d'une meilleure capacité à exprimer l'"idée " dans la peinture japonaise (ce qui va être appelé, suite à ces théories, nihonga 日本画 ${ }^{13}$ ). S'il en est ainsi, c'est bien que Fenollosa cherche plus au fond de lui à défendre une vision de l'art contre une autre, qu'à opposer une école artistique à une autre. Il y a au principe de ses arguments une théorie du bel art comme expression adéquate de ce qu’il appelle une " idée ", dont il s'efforce de faire dans certains textes comme celui-ci un usage polémique en faveur du camp qu'il a choisi ; mais la théorie elle-même n'exclut pas une interprétation universelle, puisqu'elle n'est pas par essence attachée exclusivement à l'art japonais, la possibilité étant réservée que certaines œuvres d'Occident correspondent aux critères de qualité.

12. Pour un exemple de comparaisons du premier type, voir Fenollosa 1913 : 125 ; pour le second, voir Fenollosa 1913: 82 et 85.

13. Le terme est déjà présent dans le texte imprimé du discours. 
Ce qui va dans ce sens, c'est un autre texte cette fois-ci de la main de Fenollosa et écrit dans un contexte moins directement orienté par des questions politiques, deux ans seulement après le discours retranscrit de 1882 . Il s'agit d'un compte rendu de l'Art japonais, ouvrage d'un autre japoniste, l'historien d'art français Louis Gonse (Gonse 2003, $1^{\text {re }}$ édition 1883). On peut voir cet essai ${ }^{14}$, dans lequel Fenollosa se montre parfois très virulent et sarcastique (tout en reconnaissant certains mérites à l'objet de sa critique), comme une querelle d'autorité entre deux spécialistes autoproclamés des arts japonais dans le paysage éditorial en Occident. Le nerf de l'argument de l'Américain est une critique de la hiérarchie des valeurs affichée par Gonse à l'égard des artistes japonais que celui-ci mentionne. Fenollosa lui reproche sa méconnaissance de la diversité des artistes et des filiations entre eux, ainsi que des critères proprement japonais d'appréciation des qualités picturales de leurs ouvres ${ }^{15}$. C'est ce dernier argument qui nous intéresse ici, puisqu'il est relativement nouveau dans le cas de Fenollosa, qui dans le discours de 1882 par exemple ne le soulevait pas : faute de connaissances, les Occidentaux, même des japonistes comme Gonse, ne saisiraient pas l'intérêt réel des formes artistiques qui fleurissent dans l'archipel. On retrouve la critique classique du japonisme comme une forme d'exotisme qui apprécie une œuvre étrangère en y projetant l'objet fantaisiste de ses propres attentes, risquant ainsi par là même de la condamner à l'image réductrice ainsi définie. Fenollosa, ici, se présente donc comme le connaisseur opposé au japoniste ${ }^{16}$. Or, dans le passage où il fait valoir cet argument, il se réfère à un modèle dans lequel, argue-t-il, chaque art, chaque genre artistique devrait être jugé selon les règles qui lui sont propres :

14. "Review of the Chapter on Painting in Gonse's L'Art Japonais » (Fenollosa 2009, t. I, texte $n^{\circ} 4$ ).

15. La plus ample connaissance dont dispose Fenollosa par rapport à Gonse provient en partie du fait qu'il a pu accéder au manuscrit du dictionnaire d'artistes Koga bikō 古画備考 (Documents de référence sur la peinture ancienne) d'Asaoka Okisada 朝岡 興禎, uniquement toutefois par le biais des traductions de ses étudiants, ainsi qu'aux œuvres auxquelles il a eu un accès direct plus important que Gonse, lequel se basait en grande partie sur des recueils de peintures de l'époque d'Edo (voir Marquet : 2008).

16. On pourrait évidemment contester une telle vision et dire qu'il s'agit plutôt d'un japonisme s'opposant à un autre. 
[...] this is to ignore the utter differences of the several languages in which the arts speak to us, and to have no taste for that pure expression of the peculiar innate possibilities of each, required by their several laws. [...] To admire, as typical in Eastern art, that painter whose qualities are the farthest removed from the genius of that art, being the nearest approximation to the qualities of European, is to confess one's self to have no taste for the special flavor of the former. (Fenollosa 2009, t. I, texte $n^{\circ} 4: 28$ )

On comprend l'argument de Fenollosa : chaque genre, chaque courant artistique nous parle dans un "langage " spécifique, et il importe donc de comprendre ce langage selon les règles propres ("several laws") qui le régissent, même s’il précise lui-même que ce modèle ne doit pas être pris de manière trop stricte. Par cette image linguistique, il est déjà remarquable que Fenollosa se rapproche d'une vision actuelle des arts, qui pourrait mieux correspondre par exemple à la façon dont des spécialistes d'histoire de l'art pourraient chercher à aborder des œuvres en s'efforçant de définir les codes esthétiques, culturels et sociaux présidant à leur genèse. Mais on remarque également que cette grille d'analyse implique, selon ses propres dires, de reconnaître à l'art européen ses propres " qualités ». Il s'agit d'une confirmation de la coexistence de plusieurs statuts esthétiques, plusieurs échelles de valeurs parallèles quoique correlées, dans la conception que Fenollosa se fait des arts, donc que celui-ci construit sa théorie avec en vue l'idée d'une pluralité esthétique.

Ce qui manque maintenant est un concept qui rende possible l'articulation de différents paradigmes esthétiques entre eux, tout en rattachant ce modèle pluraliste à la théorie générale définie par exemple dans le discours Bijutsu shinsetsu ou dans des textes plus tardifs. Un terme semble jouer un rôle important chez Fenollosa, tout en étant parfois difficile à définir : c'est celui de "synthèse " des formes et des éléments de l'œuvre dans la composition de celle-ci. L'hypothèse proposée ici, qui prend son sens si l'on suit la piste d'un jeune Fenollosa historiquement proche, par le biais de sa formation bostonienne, du mouvement historique dit " esthétisme ", est celle de l'influence, dans son emploi du terme, d'un critique anglo-saxon, Philip Gilbert Hamerton, qui bénéficiait d'une certaine audience dans ces milieux (Prettejohn 2007 : 32). En effet Hamerton a écrit dans la revue Fine Arts Quarterly, qui était lue en Nouvelle-Angleterre et en particulier dans les milieux d'avant-garde, deux articles sortis coup sur coup en 1864, juste avant donc que Fenollosa ne fasse ses études à Harvard, études qui 
l'ont vu suivre les cours de Charles Eliot Norton, un professeur d'histoire de l'art dont on sait qu'il était proche de l'un des initiateurs de l'esthétisme, John Ruskin ${ }^{17}$. Ces deux articles, qui se suivent, consistent pour le premier en une typologie qui oppose des artistes " analytiques " et "synthétiques ", afin, dans un second temps, d'annoncer le succès à venir de ceux du second type sur ceux du premier, par un retour d'influence. Le type " analytique " est exemplifié par les peintres préraphaélites, dont l'auteur s'efforce donc de prévoir la baisse de succès, par rapport à la montée d'artistes «synthétiques " dont l'exemple pris est... John Abbott Whistler. La référence ne peut pas ne pas être soulignée lorsqu'on connaît l'admiration que Fenollosa portait à l'œuvre de Whistler, qu'il cite régulièrement, en particulier lorsqu'il désire montrer que le type d'art qu'il prône pour l'avenir correspond effectivement à une certaine avant-garde. Hamerton associe Whistler à ce terme de "synthèse " (synthesis) parce que des tableaux comme la "dame en blanc" (Symphony in White $N^{\circ} 1$ ) seraient un bon exemple de ce procédé par lequel un peintre s'affranchirait de travailler les détails naturels afin de gagner autant en expressivité qu'en individualité du style, là où les peintres plus « analytiques » se caractériseraient au contraire par un souci du détail, comme le montre l'exemple de préraphaélites comme Burne-Jones ou Rossetti ${ }^{18}$, conformément à la définition qu'il donne de la synthèse dans son premier article ${ }^{19}$. Hamerton et Fenollosa ne sont certes pas les seuls à leur époque à se référer à cette notion de synthèse en peinture, mais à cause de la chronologie, de la conjonction en termes de scène intellectuelle et de milieux, ainsi que des artistes cités, on peut émettre l'hypothèse que Fenollosa a lu et médité ces textes, et qu'ils éclairent en retour son propre usage de ce terme.

17. Sur Norton, son lien avec Ruskin d'une part, et Fenollosa dont il fut le professeur d'autre part, voir Freedman (1990: 88).

18. Cet exemple est intéressant car ces deux peintres ne sont pas des exemples de style académique, ni de style réaliste.

19. "Synthesists find continual pleasure in observing the relations of things, but from their largeness of range they constantly miss minute truths [...]. Whenever they have to sacrifice either a truth a relation or a truth of detail they always sacrifice the detail. " (Hamerton 1864a : 238). Comparer ce texte avec celui de Fenollosa intitulé « Nature of Fine Arts 2 » (Fenollosa 2009 t. I, texte n² 21) est particulièrement parlant. 
Or, si l'on étudie le concept ainsi défini, on observe qu'il permet une théorie alternative de la beauté par rapport à celle qui pourrait être associée à l'idéal classique florissant dans l'académisme, mais qui reste en même temps ouverte de telle sorte à inclure les ouvres les plus antiques ou celles de la Renaissance les plus " authentiquement " classiques, car ne s'opposant pas à cette notion de synthèse, dans la logique de retour aux sources chère à l'esthétisme et à un John Ruskin ${ }^{20}$. Si la "synthèse " permet de mettre particulièrement en avant les artistes qui ont privilégié les audaces d'expression, développant ainsi un style individuel tout en exprimant un détachement "spirituel » vis-à-vis des vérités mondaines, ce qui est bien sûr le biais par lequel Fenollosa utilise cette notion pour justifier la valeur qu'il accorde aux grands maîtres qu'il consacre dans la peinture japonaise, de Sesshū 雪舟 à Soga Shōhaku 曾我蕭白 ou Hasegawa Tōhaku 長谷川等伯 en passant par la famille artistique des Kanō 狩野, on peut également présenter le travail d'idéalisation du corps dans la statuaire gréco-romaine comme l'élaboration d'une beauté par une certaine forme de synthèse, certes plus proche de la mimesis que la première, mais correspondant néanmoins aussi à une simplification et à une réinterprétation des formes naturelles. En effet, on trouve chez Hegel, qui est le philosophe auquel se réfèrent probablement le plus Tenshin et Fenollosa, des descriptions d'un processus similaire, que Hegel désigne comme idéalisation ${ }^{21}$. Hegel reprenait par là un thème ancien dans l'histoire de la théorie des arts occidentaux, qui est le perfectionnement de la nature par l'art (exemplifié par la théorie de l'electio), et, en lui donnant un sens à l'intérieur de son système métaphysique en le mettant en regard du concept d'Idéal, en faisait le procédé central de l'activité artistique, d'où naît le sentiment de beau par expression de l'Idéal dans la nature. Ce processus serait donc présent dans toutes les "formes de l'art ", depuis l'art symbolique jusqu'à l'art romantique, en passant par le stade d'équilibre du fond et de la forme constitué par l'art classique. En lui substituant le terme de "synthèse ", qui décrit au fond le même processus mais en valorisant

20. Plusieurs passages à travers ses écrits montrent que Fenollosa partageait la vision de John Ruskin d'une décadence de la peinture occidentale après le Quattrocento.

21. "Dès lors que ce qui est entaché par la contingence et l'extériorité dans l'existence ordinaire est ramené par l'art à cette harmonie avec son vrai concept, l'art rejette tout ce qui ne correspond pas à ce même concept dans la manifestation phénoménale, et c'est seulement par cette purification qu'il produit l'idéal. » (Hegel 1997 t. I : 208) 
des formes plus libres, plus subjectives et éloignées de la nature, Fenollosa et, après lui, Tenshin, qui connaissent bien la théorie hégélienne des formes de l'art ${ }^{22}$, entendent à la fois s'inspirer du modèle hégélien et le subvertir, en proposant une théorie esthétique à la fois globale et alternative dans laquelle l'art classique gréco-romain, encore assez mimétique quoique idéalisé, se retrouverait intégré, mais cependant moins valorisé que des genres plus synthétiques que seraient la peinture Song ou les œuvres japonaises de style chinois (kara-e) des treizième et quatorzième siècles ${ }^{23}$... Ainsi se dessinent les contours d'une théorie alternative de ce que Hegel appelait les formes de l'art, organisée cette fois-ci autour de l'axe mimesis-synthèse, et qui se retrouve dans le vocabulaire des deux hommes, qui malgré des différences de termes employés recouvre une logique identique : Fenollosa parle ainsi d'art "gréco-bouddhique " (qui correspond au classicisme oriental) de Nara, Okakura lui utilisant directement le terme de "classicisme ", et l'un comme l'autre parlant d' " art idéaliste " pour les œuvres japonaises de style chinois de l'époque Muromachi.

Fenollosa et Tenshin ont donc bâti une esthétique conçue avec pour ambition de prétendre rendre compte de l'expérience artistique en général,

22. Elle est par exemple mentionnée en japonais dans la célèbre histoire inachevée des arts et créations artisanales d'Asie orientale d'Okakura (Taitō kōgei-shi 泰東巧藝史, voir $3^{\text {e }}$ partie) : "Diviser, comme le fait Hegel, la peinture en trois catégories, symbolique, classique et romantique [...]»ヘーゲルの三区画のごとく象徵的、古典的、浪漫的というように 画する [...] (Okakura 2001b : 276).

23. Des interprétations comme celle de Takashina (1990:222) ou celle de Mori (1987: 25) font simplement de Fenollosa un néo-classique, qui réagit contre le réalisme pour promouvoir l'idéal antique à la façon de Winckelmann. L'hypothèse suivie ici implique que ces interprétations sont incorrectes au sens de réductrices (comment, s'il en était ainsi, Fenollosa puis Okakura auraient-ils pu estimer pouvoir faire sans contradiction d'artistes aussi différents de l'art grec classique que Kanō Tan.yū ou Sesshū le cœur de leur fonds d'exemples ?), mais explique également comment elles sont apparues : à cause justement de la plasticité du modèle ainsi généré par l'assouplissement de la notion d'" idéalisation ", l'art classique est bien intégrable dans la théorie, ce qui explique la proximité et l'apparence de classicisme de certains textes, en particulier le Bijutsu shinsetsu. D'autre part, il est indéniable que la théorie de Fenollosa-Okakura soit généalogiquement issue des approches d'auteurs néo-classiques comme Winckelmann ou Lessing : mais il ne faut pas pour cela oublier qu'elle s'apparente bien plus à un modernisme de la mouvance de l'esthétisme, qui promeut la recherche et l'individualité dans les formes. 
mais à l'intérieur de laquelle certains genres et écoles des arts japonais se trouvent de fait valorisés, la dimension universelle venant servir de fondement, si ce n'est d'alibi, à ce travail de valorisation ${ }^{24}$. Étudier plus en détail cette esthétique dans une perspective actuelle, afin de voir si l'on peut y trouver des éléments d'un modèle viable d'une esthétique à la fois objectiviste et pluraliste, pourrait donc passer par l'analyse de concepts comme celui de synthèse, et de la théorie des formes artistiques, des styles ainsi définie. Mais il faut aussi se demander si les termes ainsi valorisés, c'est-à-dire les genres, les œuvres elles-mêmes qui se trouvent pris pour objet, le sont vraiment dans le cadre d'une (re)reconnaissance réelle de leur spécificité.

\section{En attendant Ezra Pound? Fenollosa, la calligraphie et la peinture de lettrés}

Si Fenollosa avait pris effectivement en compte les différences de contexte artistique comme il le prône face à Louis Gonse, on trouverait chez lui des réflexions innovantes à l'égard des genres jusqu'ici peu valorisés en Occident par effet de rejet culturel, comme la calligraphie ${ }^{25}$. Trouvons-nous des développements, dans la suite de l'œuvre écrite de Fenollosa ou pouvant lui être attribuée indirectement, correspondant à l'exploitation d'une telle piste ? Dans le Bijutsu shinsetsu de 1882, on trouve bien une mention, très rapide, de l'art d'écrire comme exemple d'expression de l' "idée " (Fenollosa 1989 : 44) : ce genre est donc bien, dans ce texte, reconnu comme activité artistique à part entière. Mais comment en rend-il compte dans la suite de son ouvre?

24. Dans notre lecture, ils construisent un modèle dans lequel plusieurs paradigmes de valeur coexistent, de façon parallèle, même s'il existe des critères communs permettant de définir des œuvres de qualité à l'intérieur de ces paradigmes, transcendant ainsi ceuxci. Une autre lecture peut sans doute insister sur la valorisation de l'art "idéaliste " en insistant sur le contenu, à savoir les "idéaux » issus de la logique de la " voie du milieu » : dans ce cas, il y aurait bien un paradigme supérieur, en vertu d'un choix intellectuel. Voir Karatani (2004: 35).

25. Sur la valeur de la calligraphie et ses controverses, voir l'article de Laïli Dor, "Revaloriser la calligraphie : le rôle des expositions pendant les ères Meiji et Taishō " dans le présent numéro d'Ebisu. 
Les autres mentions de la calligraphie sont rares, mais lorsqu'elles sont présentes, elles permettent de mesurer le rapport complexe que Fenollosa entretient avec l'art d'écrire. Ainsi, en 1887, dans un compte rendu publié par le Blackwood's Edimburgh Magazine et dans lequel Fenollosa discute de l'œuvre d'un troisième amateur d'art japonais, William Anderson (18421900), on trouve l'affirmation suivante : "drawing is a branch of calligraphy $^{26}$ ", destinée, dans ce contexte, à montrer que tous les arts du Japon prennent, pour Fenollosa, leur origine en Chine. Il s'agit bien sûr d'une reformulation de la thèse de l'origine commune de l'écriture et de la peinture, présente aussi bien au Japon qu'en Chine (ch. shuhua tongyuan 書画同源) et dont l'Américain a sans aucun doute eu connaissance à l'occasion de ses études sur l'art japonais. Ces connaissances commencent, en 1887 , à être complétées par une conséquente expérience de première main d'œuvres diverses, grâce aux deux premiers voyages officiels dans le Kansai effectués par Fenollosa et Tenshin sous l'égide gouvernementale (Marquet 1999 : 143-145). Suit plus loin dans le même compte rendu une brève description de l'apprentissage des idéogrammes, quelques mots sur la façon concrète de les tracer ("light, supple and lithesome strokes »), et sur les différences de cette méthode avec l'écriture occidentale (mention de la position " pinceau droit coude levé »), et une réflexion sur les plus grandes « liberté et puissance " conférées au tracé par l'ensemble d'objets et de techniques qui constituent cet art (Fenollosa 2009 t. I, texte $n^{\circ} 5$ : 282). Pourtant, après ce paragraphe, Fenollosa retourne dans ce texte à son objet principal, qu'il partage avec celui qu'il critique ici, Anderson, à savoir "l'art pictural».

Dans la somme historique posthume de Fenollosa, Epochs of Chinese and Japanese Art (Fenollosa [1912] 1963 ; L'Art en Chine et au Japon selon la traduction française de G. Migeon, très précoce mais souvent inexacte, de 1913), publiée grâce au travail éditorial de sa veuve Mary, on retrouve à l'œuvre la même logique. L'ouvrage n'est pas exempt de citations, puisque Fenollosa mentionne le moment où la calligraphie aurait de façon décisive reçu ce qui allait devenir ses canons les plus classiques en Chine, et par là, au Japon. Le facteur en serait le développement des

26. "Le dessin est [en Chine et au Japon] une ramification de la calligraphie " (Fenollosa 2009 t. I, texte $n^{\circ} 5: 282$ ). 
instruments du calligraphe sous leur forme quasi moderne $e^{27}$ : pinceau avec son réservoir, encre, pierre et papier.

With these tools Chinese written characters became transformed from the several stages of cutting and smearing in clumsy symbolism into a pure calligraphic art where the flexibility of perfect brush stroke could unite with decorative proportioning [...]. (Fenollosa [1912] $1963: 37$ ).

Cet extrait, ajouté à ceux du compte rendu d'Anderson, montrent que Fenollosa disposait d'une vraie base théorique et historique pour parler de la calligraphie. C'est pourtant dans ce texte, qui nous livre la vision la plus détaillée (compte tenu du travail d'édition de Mary Mc Neil-Fenollosa) de ce qu'a pu être dans les détails la vision de Fenollosa de l'histoire de l'art du Japon, qu'apparaît vraiment le paradoxe : en dépit de ce passage sur l'essor de la calligraphie, celle-ci n'est à proprement parler pas représentée parmi les œuvres mentionnées au long de son ouvrage. Les quelques passages qui la mentionnent la présentent comme un complément poétique à certains styles graphiques originaux, par exemple dans le cas des œuvres de Hon.ami Kōetsu 本阿弥光悦 dont les « délicieuses fantaisies » sont, dit-il, rehaussées en « caractères d'or ou d'argent des poésies de cette calligraphie splendide et inimitable dans laquelle il était passé maître [...] ». Dans cet exemple, la calligraphie n'a clairement que le but accessoire et décoratif d'un ornement, de plus dans le cadre d'une "fantaisie ", c'est-à-dire d'une licence par rapport, on le devine, à d'autres styles plus orthodoxes comme le style des Kanō ou des maîtres de la peinture au lavis d'encre (suibokuga 水墨画), plus à même d'incarner le nouveau classicisme.

Ce faisant, l'Art en Chine et au Japon confirme ce que laissait entendre le traitement mineur de la calligraphie dans les essais et discours antérieurs de l'auteur : d'une part, Fenollosa disposait des ressources intellectuelles et théoriques pour approfondir la question de la place de la calligraphie, mais a, sans grande hésitation apparente, défini le périmètre de son objet d'étude en excluant presque totalement celle-ci. Les quelques passages chez Fenollosa qui affirment l'importance du genre ont ainsi deux effets : celui de souligner par contraste l'absence de traitement ailleurs, et celui de

27. La vision qu'avait Fenollosa d'un matériel qui n'aurait pas évolué depuis les Tang ressemble en fait à celle de R. Van Gulik. Voir Billeter (2001 : 52). 
suggérer ce qu'aurait pu être une esthétique fenollosienne qui, incluant plus systématiquement la considération du genre calligraphique en lui-même, aurait peut-être consisté à lui donner une interprétation propre, ou, encore mieux, aurait intégré un réel changement de perspective en donnant plus d'importance à l'expression artistique de la personnalité et de la vertu à travers les codes graphiques de l'écriture. Il en aurait sans nul doute résulté une remise en cause du fondement même d'une esthétique centrée sur la notion d'appréciation d'une forme pure, non informée par une connaissance du contexte, pour passer à une esthétique comme expression d'une personnalité individuelle, thème cher à Fenollosa. D'autre part, les quelques mentions directes de la calligraphie ont tendance à servir en fait dans un propos plus général qui est surtout consacré à la peinture, et l'art de tracer des caractères, s'il se voit conférer le statut d'art, sert surtout à expliquer les qualités spécifiques de l'art de tracer des lignes figuratives, dans le cadre de la peinture : il en est ainsi des deux mentions que l'on trouve dans le compte rendu de l'Art Japonais, dans lequel Fenollosa mentionne les règles du tracé apprises par le biais de la pratique calligraphique pour soutenir que Hokusai maîtrisait moins, comme le montrent ses peintures sur soie, cet art de la ligne que de plus grands (selon lui) maîtres (Fenollosa 2009 t. I, texte $\mathrm{n}^{\circ} 4: 13$ et 30).

Reste le dernier texte de Fenollosa, l'un des plus célèbres, qui pourrait passer pour sa contribution propre concernant la calligraphie : il s'agit à nouveau d'un texte posthume, Le Caractère écrit chinois, matériau poétique (Fenollosa \& Pound 1972, titre original The Chinese Written Caracter as a Medium for Poetry), édité et publié par Ezra Pound sur la base d'un manuscrit qu'il s'était vu confier par Fenollosa. Ce texte correspond-il à une révolution, opérée par l'auteur, dans la vision de l'art d'écrire ? Ici nous en appelons à une lecture précise de ce court mais influent essai, et à la nécessité de bien en distinguer interprétation et expression littérale. Il correspond en effet à une véritable déclaration de supériorité poétique, conceptuelle et de contenu du sinogramme sur l'alphabet, parce que bien sûr ces propriétés résident également dans son aspect graphique, imagé (mais pas seulement : ce sont tout aussi des propriétés grammaticales et de ce fait logiques, qui tiennent à la fois à la construction du caractère et à la composition des phrases en chinois). On peut alors considérer cet ouvrage comme instrumental dans la reconnaissance, en Occident, de l'art d'écrire et de ses spécificités. Mais la lettre même du texte ne permet pas d'en faire 
un traité esthétique de la calligraphie, ni même un essai sur la calligraphie : le Caractère écrit chinois est un traité philosophique sur le rapport entre pensée, poésie et langage écrit, doublé d'un éloge du chinois écrit et des sinogrammes courants, et de ce point de vue, de l'écriture comme expression directe et immédiate de l'activité de la pensée. Son objet est une théorie de la poésie comme rappel de la pensée originelle présente mais oubliée dans le langage courant, et de l'écriture (au sens d'un appareillage de signes écrits) comme pouvant être vecteur de cette poésie conçue comme retour à l'origine du langage, à partir de quoi les sinogrammes sont présentés comme un vecteur privilégié d'une telle activité poétique.

Fenollosa (peut-être réinterprété par Pound, génie certes bien à part ?) se situe donc ici dans la lignée d'une certaine philosophie du langage qui cherche le rapport entre celui-ci et les actions et passions premières et originelles, qu'elles soient physiques ou intellectuelles (à la manière de Locke et de Condillac et du "langage d'action " de celui-ci), et de la poésie comme rappel de ce lien perdu, dans la lignée du Rousseau de l'Essai sur l'origine des langues. C'est d'ailleurs pour ce propos, qui cherche à faire connaître une réflexion intrinsèque au langage écrit chinois, que l'ouvrage a parfois été reconnu comme porteur d'enseignements par des philosophes, par exemple Jacques Derrida (1967 : 140). Mais rien sur la pratique de l'art d'écrire en tant qu'art, ni même vraiment sur l'activité même d'écrire : pas d'historique de cette activité, pas de mention des cinq grands styles calligraphiques, pas de description de l'art de tracer, plus rien sur le lien entre matériel (pinceau, encre, papier) et conditions de l'expression que l'on trouvait dans L'Art en Chine et au Japon, rien sur le geste et l'art du geste, si cher à Jean-François Billeter, qui fait, dans son essai sur la calligraphie, du geste et de la place du corps des composantes essentielles de ce genre (Billeter 2001 : 63-65, 85-88, et voir les chapitres VI, VII et VIII passim). Certes, faire comme Fenollosa l'éloge de l'expression graphique de la pensée dans le sinogramme débouche implicitement sur l'idée de son exceptionnel potentiel artistique, aussi bien en lui-même (le sinogramme comme forme, dans sa variété plastique et expressive) que dans son pouvoir d'engendrer de nouvelles formes - que l'on pense aux exemples de calligrammes véritables (figures de personnes dont la silhouette provient d'un sinogramme ou d'un hiragana) dans l'art japonais, que Fenollosa connaissait peut-être. Mais alors, pourquoi ne pas avoir souligné un tel rôle dans la lettre même du texte, de la part d'un écrivain attaché par 
ailleurs à défendre non pas la pensée ou la poésie, mais bien les arts plastiques d'Asie orientale? Quoiqu'il en soit de l'implicite, la lettre même du texte semble indiquer qu'il fut conçu avec en vue un sinogramme certes écrit, mais somme toute assez dématérialisé, sans expliquer les conditions de son tracé et de son apprentissage, à la façon dont on a pu dire que l'approche esthétique dématérialisait les œuvres.

Dans le doute et en se basant sur les seuls écrits publiés, il faut en rester au constat du contraste entre des passages qui laissent entendre clairement que Fenollosa avait le potentiel d'accorder une vraie place à l'art d'écrire, et l'absence de traitement qu'il lui réserve dans sa description effective des arts. Ce contraste est, comme on l'a vu, répété, et présent aussi loin qu'en 1887 (dans le texte sur Anderson) : il semble donc dénoter une structure intellectuelle, une position donnée, et non être le fruit des conditions. Est-ce la conscience de son manque d'expertise dans un domaine qui aurait supposé une pratique dont il ne disposait pas ? Il est vrai que Fenollosa a été plus volubile sur les arts dont il avait une pratique directe, comme le nô, mais cela n'est pas vrai jusqu'au bout, puisqu'il s'est exprimé abondamment sur la peinture à l'encre, dont il avait une connaissance bien plus théorique que de la peinture à l'huile qu'il avait pratiquée dans sa jeunesse dans le Massachusetts. Si Fenollosa avait été borné par des scrupules concernant sa propre érudition, dont il connaissait sans doute les limites, il aurait moins écrit, ou différemment, de façon moins audacieuse, et aurait été d'ailleurs de ce fait bien moins influent. Il faut donc chercher ailleurs la raison de son silence : c'est que l'art d'écrire est bel et bien « hors-cadre » pour Fenollosa, pour reprendre l'expression de Michael Lucken ${ }^{28}$, mais sans doute non pas tant, comme le dit Doris Croissant, parce qu'il « ne pouvait pas être concilié avec l'idée hégélienne de la peinture ${ }^{29}$ ", étant donné que Fenollosa a montré avec Tenshin, selon l'hypothèse de notre première partie, une capacité réelle à s'éloigner de la lettre hégélienne dès qu'il jugeait cela conforme à ce qu'il percevait de l'art à partir de ce qu'il ressentait comme étant la

28. Lui-même l'emploie pour expliquer l'absence d'enseignement de cet art à l'École des beaux-arts de Tokyo (Lucken 2001 : 37).

29. "In Bijutsu shinsetsu Fenollosa had avoided referring to calligraphy, probably because it could be reconcilied neither with the Hegelian "idea of painting" nor with his own dislike of literati paintings for their adherence to the canons of poetry. " (Croissant $2006: 165)$. 
supériorité de la peinture japonaise. Mais n'est-ce pas encore précisément de ce projet de mettre en évidence cette supériorité, cette valeur artistique, que naît paradoxalement une telle discrimination?

Si l'approche des arts chez Fenollosa est esthétique, c'est peut-être à l'origine à cause de son éducation, mais aussi et surtout parce qu'elle se veut universaliste, dans une conception de l'" universalisme " très concrète, qui se base certes sur la notion d'une finesse de l'âme devant reconnaître la valeur de l'œuvre, mais sans plus d'éducation que celle la conduisant à cette finesse, et certainement dans un rejet emersonien de toute érudition excessivement élitiste. Concrètement, Fenollosa veut qu'une œuvre de "fine art " puisse être appréciée par toute personne ayant bénéficié d'une éducation moyenne, ses concitoyens de Salem et en Nouvelle-Angleterre, les amateurs de japonisme à New York ou à Paris. Or, comme lui-même se considérait sans doute comme un bon sujet-test, il n’a jamais pris vraiment la peine de mesurer la particularité de certains de ses goûts. Ce dernier détail se voit dans sa critique d'un autre art, la peinture de lettrés ou bunjinga 文人画, au sujet duquel nous renvoyons à d'autres analyses ${ }^{30}$ : il y a néanmoins un fort parallèle avec le cas de la calligraphie, quoique celle-ci soit simplement négligée là où la première est nettement dépréciée. Dans les deux cas, il s'agit en effet de genres d'œuvres dont il est sans doute plus évident que pour d'autres genres, y compris pour Fenollosa, que l'appréciation de leur qualité passe par une connaissance éprouvée du contexte, et de certaines règles qui, de plus, changent selon les époques, les cercles de composition ou encore les théoriciens. Le problème n'est-il pas au fond, dans un cas comme dans l'autre, que Fenollosa renonce à les faire entrer dans le cadre de ce que l'on pourrait appeler le point de vue esthétique sur l'art, c'est-à-dire la volonté de rendre compte de la valeur artistique du point de vue d'un spectateur idéal, universel, qui est le sien parce que c'est par là que, pour lui, doit passer la reconnaissance de l'art japonais au niveau mondial ? Il met ce faisant en lumière un défaut possible de ce point de vue, à savoir la tendance, dans

30. L'analyse de l'auteur du présent article, qui sous-tend celle-ci, sur la question du bunjinga figurera dans l'ouvrage collectif Japon Pluriel 9 (à parâtre, éd. Picquier). Pour des références à disposition, voir surtout Yamaguchi (1993), auquel on peut ajouter Guth (2006) et Croissant (2006: 165, voir note précédente), à ceci près que la vision chez ces deux dernières d'un Fenollosa simplement dépassé par les enjeux de sa propre position peut sembler contestable, si l'on suit les arguments présentés ici. 
l'idée de garantir une telle universalité, d'associer au fond toujours l'idée de valeur artistique à des œuvres dont on pourrait croire (à tort ou à raison) que leur valeur dépend au fond d'une qualité formelle, perceptible par tout spectateur nonobstant sa connaissance du contexte de genèse de l'œuvre.

Il ne s'agit pas ici de sous-entendre qu'il y aurait une valeur absolue de la calligraphie en tant qu'art qui aurait attendu pour ainsi dire de toute éternité, et que Fenollosa n'aurait pas su voir : comme dans la traduction elle-même de sho 書 (à laquelle Billeter par exemple préfère le terme d'" art d'écrire ", que nous avons beaucoup employé ici), il ne va pas de soi de faire de celle-ci un "art ", sans que cela ne change le sens même de ce terme par rapport à d'autres usages auquel le monde des arts conservés dans des musées, ou encore des arts contemporains, nous a habitués. Il s'agit en revanche de remarquer que Fenollosa n'a que très peu creusé cette piste de l'art calligraphique, alors même qu'il avait dans sa théorie des éléments allant dans ce sens, et que le déséquilibre de fait qui en résulte, en termes de défaut d'exposition de ce genre dans ses textes, est à son tour significatif concernant cette théorie. Le contexte historique de l'ère Meiji peut également être invoqué : reconstruire l'idée que l'art d'écrire pouvait prendre sa place au même titre que d'autres formes de création, sur le même plan, n'allait certes pas de soi pour les contemporains eux-mêmes, comme le montre la polémique entre le peintre Koyama Shōtarō 小山正太郎 et Okakura au début des années 1880 ; il fallait réexhumer les œuvres, les notions, les textes ; la " tradition ", selon la logique que l'on connaît bien depuis Hobsbawm et Ranger, restait entièrement à inventer. Ce sont pourtant Okakura et Fenollosa eux-mêmes qui se sont fait forts de faire reconnaître en tant que beaux-arts des arts non encore reconnus, et d'ouvrir le champ de leur étude historique moderne ${ }^{31}$; Fenollosa apparait donc ici quelque peu en porte-à-faux avec ce programme.

Il faut enfin rappeler que le faible volume des mentions n'exclut pas, à titre purement théorique, de déduire à partir des quelques passages cités, ainsi que des thèses esthétiques générales de Fenollosa, ce qui pourrait chez lui être vu comme une théorie latente de l'art d'écrire : soit en mettant l'accent sur la thèse de l'œuvre d'art comme expression réussie d'un sujet,

31. Pour un bilan critique de la méthode historiographique de Tenshin, de ses apports, de ses limites et de son rôle en tant que jalon, voir Inaga (2001b). 
dans le double sens d'un thème et de l'auteur de l'œuvre (sentiment ou état d'esprit, suite de pensées, valeur morale, sociale ou religieuse d'une personne) par une forme dotée elle-même des qualités aptes à exprimer celui-ci, soit en s'inspirant de l'aspect plus formel de son esthétique, qui insiste sur la triple qualité de cohérence quasi organique, d'harmonie (" gradation et contraste ») et de singularité absolue de la forme belle. Développer cet aspect formel pourrait aussi passer par une exploitation du rapprochement de la musique et de la calligraphie, analogie qu' explore et affectionne Billeter tout en en montrant les limites (2001 : 88-96) : Fenollosa, qui a grandi dans un milieu de musiciens, se sert lui-même souvent, en effet, de l'exemple de la musique pour expliquer ses idées; si bien que l'on pourrait même être tenté de dire qu'au fond Fenollosa analyse tous les arts sur le modèle d'une composition musicale, l'« idée », forme auto-suffisante tout en étant expressive d'un idéal ou d'un sentiment, se caractérisant par une individualité absolue, à la façon dont chaque mélodie est à la fois cohérente et unique, et pouvant renvoyer au fond à la composition musicale comme à son modèle.

Mais puisque ce point de vue du spectateur universel, par lequel nous avons défini l'esthétique, est précisément celui pour lequel nous avions proposé de rechercher un modèle viable, la façon dont Fenollosa se met ici en contradiction avec la règle qu'il énonçait dans le texte sur Louis Gonse, précisément à cause du point du vue esthétique qui est le sien, tendrait à révéler un défaut intrinsèque de cette approche en général. Un tel écueil pourrait-il être évité ? On peut en douter, puisque la logique même consistant à poser des valeurs esthétiques universellement transmissibles semble devoir provoquer ces effets de perspective par lesquels certains genres sont considérés comme artistiques et d'autres non, et que les analyses précédentes ont tendu à le confirmer. Peut-être, après avoir distingué comme Yves Michaud esthétique et poïétique, horizon de réception et univers de la création, faut-il statuer qu'une théorie des arts basée sur le premier ne peut jamais fonder un discours sur les œuvres, mais ne peut parler que d'elle-même ? À moins qu'il soit possible d'éviter cela en élaborant encore un autre modèle. Mais comment et lequel ? 


\section{Okakura Kakuzō : vers un autre modèle esthétique ?}

Analyser en détail les différentes façons dont on peut observer Okakura répondre à ce problème du point de vue esthétique, point de vue qu'il a fait sien au moins pour une partie de sa carrière, dépasse le cadre du présent travail ; d'autant qu'il s'agit d'un penseur fuyant, dont l'évolution est complexe. Mais nous voulons terminer en présentant une hypothèse de lecture selon laquelle cette évolution complexe de "Tenshin » à travers ses textes s'expliquerait en partie par sa volonté de répondre au problème de l'esthétique. Il s'agit de chercher dans ses textes des éléments de réponse à cette question.

Okakura a longtemps eu de nombreux points communs, dans sa façon d'aborder l'esthétique, avec Fenollosa, y compris dans ses travaux pionniers d'histoire de l'art. On a vu en effet qu'il se servait comme celui-ci du modèle alternatif des formes de l'art présenté en première partie. C'est le cas notamment dans son essai historiographique en anglais Ideals of the East (Les Idéaux de l'Orient, 1917) : jusqu'à 1903 inclus donc, on peut le considérer comme l'autre grand utilisateur de cette théorie, malgré des divergences notoires. Or, on peut remarquer qu'à cette proximité a fait écho une attitude somme toute similaire à celle de Fenollosa vis-à-vis de la calligraphie.

Okakura a pourtant commencé en jouant un rôle important dans l'histoire de la réception moderne de ce genre, par le biais de sa participation à la controverse avec le peintre occidentaliste Koyama Shōtarō ${ }^{32}$. Si le futur "Tenshin » argumente alors contre le déni d'appartenance de la calligraphie aux «beaux-arts » (bijutsu美術), il ne définit en fait vraiment dans ces textes ni la teneur exacte du caractère artistique propre à la calligraphie, ni (ce qui est conjoint) la nature de ce que pourraient être les «beaux-arts " pour que la calligraphie y soient incluse. Le lecteur semble laissé libre de remplir lui-même la signification d'une telle notion. Ce choix rhétorique, défendable en lui-même, laisse toutefois une impression d'impuissance théorique, dans ce texte, de la part du jeune Kakuzō, en dépit d'une forte intuition de son objet. Cette impuissance ou ce refus initial de donner une

32. Sur la polémique Koyama-Okakura, ainsi que pour ses références bibliographiques, nous renvoyons à l'article de Laïli Dor dans le même numéro. 
définition sembla de plus présider à son rapport avec ce genre par la suite : Okakura a défendu le parti de l'utilisation du pinceau et de l'encre dans l'enseignement général, tandis que les cursus de formation au nihonga, qu'il contribua largement à mettre en place à l'École des beaux-arts de Tokyo et l'Institut des beaux-arts du Japon, reposaient sur l'enseignement d'une posture de travail identique à celle enseignée aux apprentis calligraphes $^{33}$. Et pourtant, il n'y eut pas sous sa férule d'enseignement d'art calligraphique dans ces établissements... De plus, dans les ouvrages historiques de la période intermédiaire, Les Idéaux de l'Orient de 1903 ou les cours en japonais parallèles à cet ouvrage (et regroupés aujourd'hui dans la première partie du volume intitulé Nihon bijutsu-shi 日本美術史, 2001a), la calligraphie est également profondément sous-représentée : elle ne compte pas vraiment dans l'ossature de la périodisation historique et stylistique, et les mentions en sont très rares. Celle que l'on trouve dans Les Idéaux de l'Orient est la plus intéressante, parce qu'elle donne la meilleure approximation d'une spécificité du genre :

Le culte de la calligraphie qui atteint, pour la première fois, un grand développement pendant cette période laoïste, est purement et simplement le culte de la ligne. Chaque coup de pinceau porte en lui son principe de vie et de mort, lié aux autres lignes, il crée la beauté d'un idéographe [sic]. Il n'en faudrait pas déduire que la perfection d'une grande peinture chinoise ou japonaise réside uniquement dans l'expression et dans l'accent des traits et des contours, mais ceux-ci possèdent par eux-mêmes, en tant que lignes, une beauté abstraite qui leur est propre. (Okakura $1917: 70)$

Tenshin présente ici la calligraphie dans la foulée d'une description de la peinture, qui s'est développée selon lui à la suite des canons définis par le maître des Song du Nord Guo Xi 郭熙, auteur du célèbre traité sur l'art du paysage Linquan gaozhi 林泉高致 (Haut Message des forêts et des sources). Mais le propos dans ce passage est flou, car si la beauté des idéogrammes

33. En vertu de la thèse de l'identité originelle entre peinture et calligraphie ; il s'agit de la posture kenwan chokuhitsu 懸腕直筆 que l'on pourrait traduire par " pinceau droit, coude levé ", et que nous avons déjà mentionnée. C'est le peintre Hashimoto Gahō 橋本雅邦, qui eut un rôle majeur dans la définition des premières orientations du nihonga, qui insista sur son importance (Weston 2004 : 89-90, et voir en général 75-107 sur le cursus instauré par Okakura). 
en eux-mêmes est reconnue dans la seconde phrase, l'expression qui suit de " beauté abstraite ", dans la troisième, est, à suivre la lettre même du texte, attribuée aux " traits et contours" (outlines and contours) des formes figuratives de la peinture. Le lecteur pourrait être tenté d'inférer que ce qualificatif d'“ abstraite " s'applique également à la beauté des caractères, puisque la calligraphie est présentée dans une continuité avec l'art de la ligne à l'encre picturale, dont elle serait une sorte de pratique pure ("culte ») réalisée par le truchement de l'inscription d'un texte. Mais la formulation ne permet pas, à rigoureusement parler, une telle inférence, et demeure donc ambiguë, la nature exacte de la teneur artistique de la calligraphie demeurant incertaine en dehors de l'attribut de " beauté ". Pour résumer, on peut soit risquer l'interprétation d'une valeur des calligrammes résidant dans leur capacité de faire éclater cette " beauté abstraite " à l'état pur, soit simplement considérer qu'il n'y a pas assez d'éléments pour affirmer cela. Quoi qu'il en soit, la calligraphie tend ici à nouveau à être traitée comme une adjonction ou une propédeutique à la peinture, comme dans la formation de l'École des beaux-arts de Tokyo ou dans la critique de Hokusai (et de Louis Gonse) dans le texte de Fenollosa de 1884, malgré la reconnaissance nominale d'une valeur propre.

L'impression est donc à nouveau celle, comme pour Fenollosa, d'une piste ouverte mais non exploitée, là aussi pour cause de difficulté à trouver un statut dans un cadre esthétique à un art qui dépasse ce cadre. Mais la différence est que chez Tenshin, sans doute bien plus conscient du problème, on peut remarquer une tentative de faire évoluer le cadre luimême, en remettant en cause la distinction entre beaux-arts ou fine arts d'une part, et arts considérés jusqu'ici comme mineurs, artisanat et arts " décoratifs " d'autre part. Pourtant, nous voudrions observer, sur cette question qui demanderait un traitement bien plus détaillé, que le travail de Tenshin en ce sens, tout en ayant une importance historique considérable, correspond en fait à des avancées très mineures sur le plan conceptuel, comme sur le plan concret. Dans un passage célèbre du manuscrit des notes d'un cours qu'il donna en 1910 à l'université impériale de Tokyo (Okakura 2001a : 404), et qui fut par la suite (à l'occasion des premières œuvres complètes) publié sous le titre de Taitō kōgei-sh $i$ 泰東巧藝史 (Histoire de la création artistique en Asie orientale; publié avec ses cours plus anciens dans Okakura 2001a), Okakura Kakuzō explique qu'il utilise volontairement ce néologisme de kōgei pour définir le périmètre de 
son étude ${ }^{34}$. Il l'a formé de $k o ̄$ 巧 (habileté technique) et gei 藝 (technique, talent), afin d'éviter toute distinction problématique entre de supposés beaux-arts et les autres créations artisanales. Il s'agit bien sûr de ne pas poser entre certains genres et d'autres une distinction artificielle et surtout discriminante, qui pourrait nuire à la pleine reconnaissance des qualités de l'art japonais ancien et " authentique ». Ce qui prépara une telle révolution de sa conception de l'historiographie fut la participation d'Okakura, en tant que membre consultatif, à la Commission impériale pour l'Exposition universelle de Paris de 1900, au cours de laquelle le Japon avait assumé une telle redéfinition du périmètre des arts " nobles ", comme il apparaît dans le catalogue publié en 1901, Histoire de l'art du Japon, à la rédaction duquel Tenshin a été un temps associé, notamment à travers la valorisation des objets conservés dans le Shōsōin 正倉院, le trésor impérial de Nara. Okakura n'est certes pas le seul à avoir initié ce mouvement : on peut tout autant en attribuer l'initiative à celui qui fut son supérieur hiérarchique, Kuki Ryūichi 九鬼隆一, le directeur du Musée impérial, ou encore à l'artisan plus constant du projet, l'historien Kurokawa Mayori 黒川真頼, luimême auteur en 1878 d'une Histoire des arts manuels du Japon (Kögei shiryō 工藝志料). Cette problématique a conditionné la participation artistique du Japon aux expositions universelles pendant deux décennies, et Okakura n'en fut qu'un des contributeurs. Sur le plan patrimonial en tout cas, il fit suivre ses propos d'actions, puisqu'il organisa au cours de son travail d'assistant conservateur du Musée des beaux-arts de Boston plusieurs expositions d'objets japonais qui avaient pu être qualifiés jusqu'alors de décoratifs : inrō 印籠, tsuba 鋁 et montures de sabres, laques. Il accompagna ces expositions de textes historiques fournis, montrant son intérêt concret pour ces genres. Pourtant, on peut remarquer que ces textes demeurent isolés, donnant l'impression qu'avant Taitö kögei-shi, ils sont de fait sur un autre plan que la peinture, la sculpture et l'architecture, qui font le " gros » des exemples

34. "Cependant, dans ce cours, le terme de beaux-arts n'étant pas vraiment approprié, nous emploierons ceux d'art ou de créations de l'art et de l'artisanat afin de couvrir plus convenablement le champ de nos recherches. »けだし本講に美術の語はやや妥当ならず、藝 術または巧藝の語を用いるをもつて適当とせる範囲をも包含す (Okakura 2001a : 264). Il est difficile de traduire un tel passage en raison des nuances apportées par les mots composés, ainsi que des différences culturelles que le vocabulaire des deux langues respectives a fixées en les substantialisant. 
de son ouvrage Les Idéaux de l'Orient. De plus, dans le célèbre Livre du thé de 1906, qui peut être vu à cet égard ici comme un ouvrage de transition (dans l'histoire de la reconnaissance des genres « décoratifs » mais aussi dans l'évolution d'Okakura lui-même), on trouve bien quelques mentions des artisanats liés aux ustensiles de thé dans le dernier chapitre, « les maîtres de thé », mais le propos s'appuie encore beaucoup sur des œuvres tirées de la peinture, de la musique ou de l'architecture. Si bien que la redéfinition par Tenshin du périmètre des arts demeure essentiellement programmatique, et peu suivie d'effet théorique réel, si on la compare aux écrits et à l'action d'un penseur comme Yanagi Sōetsu 柳宗悦 (1889-1961) par exemple, qui à partir des années 1920 entreprendra une véritable réévaluation des " arts populaires ${ }^{35}$. Il y a certes une différence de génération entre eux, Yanagi, qui écrit deux décennies après Tenshin, bénéficiant du travail préparatoire fait par ses prédécesseurs, et de l'évolution des données culturelles ; mais comparer les bilans des deux théoriciens de l'art n'en demeure pas moins significatif sur les limites rencontrées par Okakura ${ }^{36}$.

La redéfinition du périmètre et de la hiérarchie des arts peut néanmoins n'être considérée que comme un angle d'attaque possible du travail d'adaptation de la théorie esthétique aux arts japonais : un autre angle consiste à travailler sur le cœur même de la théorie, à savoir le jugement d'appréciation artistique lui-même. C'est ainsi que nous proposons de lire l'un des tout derniers textes de Tenshin, Nature in East Asiatic Painting. Dans ce texte issu d'une conférence donnée au Musée de Boston en 1911, deux ans avant

35. Sur Yanagi, voir le numéro spécial L’invention des arts populaires. Yanagi Sōetsu et le Mingei de la revue Cipango. Cahiers d'études japonaises (Marquet \& Butel 2009) et l'article de Damien Kunik dans le présent numéro d'Ebisu.

36. Il faut citer l'analyse d'Inaga pour qui la valorisation de l'école Rinpa 琳派 par Okakura lorsqu'il essaye de définir le programme du nihonga à venir implique une certaine forme d'acceptation des caractères pouvant être jugés, selon cette catégorie dirimante à l'époque, les plus « décoratifs » dans les arts plastiques japonais, parce que c'est ainsi que le voyaient certains Occidentaux comme par exemple Duret, et qu'Okakura lui-même percevait l'œuvre d'artistes comme Ogata Kōrin ou Hon.ami Kōetsu (voir Inaga 2001a : 74) comme précurseurs à l'égard des impressionnistes. Il faudrait donc voir dans ce rapprochement avec les avant-gardes une autre forme de remise en cause du paradigme des beaux-arts, mais si on ne peut que suivre une telle analyse, il faut remarquer que ce rapprochement demeure modeste à l'intérieur des hiérarchies de valeurs entre artistes et écoles généralement posées par Okakura. 
sa mort, celui-ci explique que ce n'est pas le beau qui serait la catégorie centrale de l'esthétique orientale, mais l'« intéressant " (interesting) : "Another point of distinction about Eastern art, is that it is not interested in beauty as such. The quest of art is not the beautiful but the interesting. " (Okakura 1984 t. II : 148). En un sens, on peut dire que ce déplacement des termes de l'esthétique pourrait sembler signifier un renoncement à celle-ci dans sa figure universaliste, puisque l'idée de rassembler sous un même principe des expériences hétérogènes de plaisir artistique paraît abandonnée. Pourtant, Okakura continue à s'adresser, dans ce texte, à une audience étrangère, en l'occurrence américaine, bostonienne et constituée de bourgeois plus que de spécialistes : la conférence est ouverte à public non érudit, elle suit un objectif de vulgarisation. Il ne perd donc pas sa croyance dans la possibilité d'un amateurat étranger, éclairé sans être nécessairement érudit. En effet, son propos refuse moins à une telle audience la possibilité d'accéder à l'art " oriental ", faute d'une connaissance adéquate qu'elle aurait à acquérir, qu'il ne s'efforce au contraire d'en livrer les clefs en quelques pages, ce qui suppose bien une base anthropologique commune. N'observe-t-on pas ici une tentative de sa part de conserver l'aspect universel de jure de l'esthétique, tout en proposant une ouverture de facto de sa pratique à l'intégration de paramètres contextuels déterminés, et ce en proposant une plus grande plasticité des critères d'appréciation? Il faut en effet remarquer que ce terme d' "intéressant " permet de maintenir le jugement esthétique ancré dans une forme ou des percepts, tout en ouvrant le champ des sentiments éveillés par l'objet, et donc le champ des expressions artistiques ${ }^{37}$. D'autre part, un élément important de ce texte est que le terme de beauty y est également présent, en contradiction apparente avec la citation ci-dessus :

The beauty of draughtsmanship [in Eastern painting] lay not only in the power of actually drawing the object, but also in the abstract beauty which lay in the power of the brush stroke. (Okakura 1984 t. II : 150)

37. C'est peut-être par là que ces tâtonnements d'Okakura rejoignent des démarches esthétiques contemporaines comme par exemple celle de F. Flahault qui élabore une théorie de l'art et de sa réception dans la lignée du jeu enfantin (Flahault 2003), ou celle de J.-M. Schaeffer et ses recherches sur la conduite esthétique comme émission et réception de "signaux à coût élevé ", dans la mesure ou l' " intéressant » renvoie à un accroissement de l'attention (Schaeffer 2003). 
Il nous semble que cette entorse à son propre propos s'explique par le fait que Tenshin se place dans cette phrase du point de vue de son audience américaine. Si l'on cherche à reconstituer la logique sous-jacente, on peut reformuler le propos ainsi : les arts d'Asie orientale s'expliquent mieux du point de vue de l'appréciation formelle par une catégorie comme l' "intéressant ", mais on peut aussi convoquer la notion de beauté pour les aborder, quoique cette notion soit moins adéquate et doive être acclimatée $^{38}$. Selon une telle optique de lecture, Okakura ne rejetterait pas tant le point de vue esthétique qu'il s'efforcerait de le faire évoluer depuis l'intérieur, en intégrant dans la réflexion esthétique une conscience des limites de celle-ci ; et pour cela, il proposerait une nouvelle forme de théorie syncrétique où coexisteraient de façon problématique (et non plus syncrétique) différents critères, ceux-ci assurant une plus grande ouverture du champ d'objet.

Selon cette piste de lecture, "Tenshin » Okakura Kakuzō aurait été, dans la décennie 1900-1910, en quête d'un nouveau modèle esthétique visant à dépasser les problèmes de celui qu'il élabora auparavant avec son maître Fenollosa, évolution arrêtée net lorsqu'il fut fauché par la maladie, à l'orée de la cinquantaine. Cette évolution fait apparaître le chef-d'œuvre de Tenshin, le Livre du Thé, comme un ouvrage de transition avant son chant du cygne de 1911, parce que le changement de perspective sur l'esthétique commencerait à s'y faire sentir. On peut en effet remarquer qu'à un changement de ton certain de l'auteur dans cet ouvrage, correspond également une très nette apparition de la thématique du doute vis-à-vis de la capacité de ce qu'il appelle "les deux moitiés du monde " à se comprendre mutuellement. Pourtant, là encore, Okakura s'adresse en anglais à un public occidental, si ce n'est mondial, et continue à se présenter en passeur de connaissances littéralement étrangères à des non-Japonais, mais qui font signe vers des facultés communes à l'humanité dans son ensemble. Si certains passages insistent sur les obstacles locaux à la compréhension mutuelle, qui ne peuvent manquer d'apparaître à partir du moment où l'auteur ouvre sciemment l'œuvre d'art à son environnement, dans toutes les acceptions : à la fois son extérieur, ses relations avec son environnement

38. On observe dans cette phrase que ressurgit cette catégorie d'" abstraction ", que Kakuzō avait initialement convoquée pour la calligraphie. 
dans l'espace (la nature autour et dans la pièce de thé), et son contexte, littéraire, intellectuel et poétique, en revanche, Tenshin semble vouloir maintenir l'idée d'une accessibilité du sens de l'œuvre à un public non averti, voire universel, comme on peut par exemple voir dans l'exemple de la traversée du roji 路地, le chemin menant à la pièce de thé, par l'hôte :

Quiconque a foulé le sol de l'allée qui traverse le jardin ne peut manquer de se rappeler combien son esprit s'élevait au-dessus des pensées ordinaires, tandis qu'il marchait dans la pénombre crépusculaire des arbres à feuilles toujours vertes [...] (Okakura $2000: 73)$

Le terme de "quiconque " implique bien une universalité de l'effet esthétique produit par cette traversée. Dans la suite, le court exercice herméneutique du vocabulaire esthétique de l'architecture de la chambre de thé auquel Tenshin se livre montre une esthétique bien plus essentiellement axée sur la transmission d'un sens que sur l'épanouissement d'une forme exprimant ce sens. Les qualités esthétiques destinées à exprimer, selon son interprétation, les "idéaux zen " peuvent décidément éluder l'observateur non averti. Mais elles n'en expriment pas moins ces idéaux de façon immanente : la simplicité des décorations pour la pureté du cœur ; le caractère réduit de certaines proportions pour " inculquer " l'humilité (Okakura 2000 : 75) ; la propreté comme expression du soin et de l'application de l'esprit (Okakura 2000 : 77), application elle-même insuffisante sans une recherche de la fantaisie qui doit exprimer l'individualité (Okakura 2000 : 78), expression nécessaire de la vitalité et de la liberté qui doivent s'épanouir dans l'art comme dans la religion ; l'asymétrie enfin, afin d'exprimer la vanité de la recherche de toute perfection en ce monde (Okakura 2000 : 83-84). Chez Okakura, jamais autant que dans cet ouvrage la tension entre un modèle centré sur un spectateur idéal et universel, et la conscience des contresens existant entre les différents paradigmes culturels, n'a été aussi forte : mais cela demeure une tension plus qu'une rupture, parce qu'il cherche encore à concilier les deux dimensions. 


\section{Conclusion}

L'esthétique développée dans les décennies 1880 à 1900 par Fenollosa et par Okakura correspond bien à un modèle universaliste qui a intégré l'idée d'une pluralité des paradigmes artistiques, articulée autour du concept de synthèse. Mais ce modèle présente aussi des contradictions, même s'il renferme certainement des idées et des enseignements qui mériteraient d'être plus étudiés sous une telle optique. Ces contradictions pourraient conduire à rejeter le paradigme esthétique en général, en tant qu'il serait grevé d'un défaut intrinsèque, conditionné par sa nature de point de vue axé sur la réception indéterminée et non informée ; ou du moins, conduire à ne pas accorder à ce point de vue un statut autre que superfétatoire, celui d'une grille de lecture plaquée sur les œuvres d'art sans jamais pouvoir être considérée comme objectivement pertinente. La tendance de l'esthétique universaliste à privilégier la forme sur le fond peut certes sembler évidente, de même qu'est connu le procédé de renversement des valeurs par lequel Okakura a proposé dans le Livre du Thé, en montrant la grandeur des idées exprimées par un genre et des objets (les arts du thé) en apparence modestes, une véritable refondation de l'échelle des valeurs éthico-esthétiques. Mais ce qu'il faut remarquer, c'est qu'au principe de toute redéfinition du périmètre des beaux-arts, de toute revalorisation d'arts jusqu'alors qualifiés de " décoratifs ", Okakura a en fait jugé nécessaire de travailler sur le cœur même du paradigme esthétique, c'est-à-dire la théorie du jugement d'appréciation. Il l'a fait tantôt en assouplissant les termes utilisés pour décrire la " relation esthétique » entre le spectateur et l'œuvre, par l'utilisation d'expressions renvoyant à l'intérêt, l'étonnement et la découverte, tantôt en cherchant à définir une esthétique dans laquelle la notion de "beau " peut toujours être convoquée, mais dont le vrai cœur est une conception de l'art comme communication d'unités de sens et de valeurs au moyen des formes. Mais aussi par la relation de ces formes les unes avec les autres (architecture de la sukiya 数寄屋, jardin et nature autour, rôle joué par la saison et la référence à celle-ci dans le poème choisi, etc.) - dépassant donc la conception d'un beau comme attribut d'un objet isolé.

C'est en effet en redéfinissant le principe (la théorie du jugement esthétique) que l'on peut efficacement déterminer ensuite le champ d'objet, à savoir le périmètre des arts, des œuvres dotées de valeur artistique, plutôt que l'inverse. Conserver l'idée d'une classe d'objets dotés d'une valeur pour 
la société était le but ultime de "Tenshin » comme celui de Fenollosa. La difficulté à laquelle ils se sont heurté, au fond, a été de vouloir conserver cette notion de valeur sociale inhérente à la notion de beauté tout en la séparant du formalisme de celle-ci, qui tend toujours in fine à l'attacher à certaines formes plutôt qu'à d'autres, mais tout en conservant aussi le caractère universellement manifeste, perceptible de la valeur ainsi définie. Okakura estima finalement réussir cette gageure en posant l'idée (et en décrivant des genres supposés en constituer un exemple, comme ceux associés au thé) qu'était possible une esthétique de la façon dont les formes collaborent à tracer un idéal transmissible par delà les différences culturelles, sans que le sentiment de "beauté " suscité par cet idéal ne doive nécessairement pour cela être attaché à une forme unique, isolée, détachée de son environnement.

Quoi que l'on pense de la pertinence de cette hypothèse, il faut cependant remarquer que d'une part, son enseignement est qu'un plaisir esthétique peut correspondre à la saisie par le biais de formes d'un contenu de valeur culturelle sans que cela implique la position d'un beau défini comme perfection d'une forme isolée, et que l'on peut, sur le plan théorique plus général, séparer le champ de la théorie esthétique, au sens d'appréciation globale de l'art fondée sur des percepts, de celui de l'expérience du beau, tout en continuant à référer ponctuellement à celui-ci. Ce sont deux champs distincts, même si leur intersection existe sans doute, et a pu être historiquement valorisée, en particulier (seulement ?) en Occident. D'autre part, cette évolution finale de la pensée d'Okakura revient en pratique à mettre en lumière les obstacles locaux à la compréhension, et donc à réintroduire un certain relativisme de fonctionnement dans la théorie universaliste. Lui-même le savait, comme le montrent les passages les plus sceptiques du Livre du Thé, en particulier le premier essai, "La Coupe de l'Humanité ", qui est une sorte d'introduction, parfois amère, au sentiment au sens actif et concret, et non pas seulement théorique, de l'altérité. Le seul universalisme concevable, pour Okakura Kakuzō, en matière d'art, implique non seulement une exigence de reconnaissance de cette altérité (comme chez Fenollosa), mais de plus, une certaine qualité humaine d'imagination apte à saisir celle-ci, et partant, une revalorisation du sentiment, qui est le matériau aussi bien que le stimulus de cette imagination, dans l'esthétique, à l'instar de chez Rousseau qui suit un chemin en cela similaire. Il implique en fait une philosophie de la volonté. 


\section{Bibliographie}

ANDERSON William, [1886] 2007

Pictorial Art of Japan, 3 vol., Tokyo, Synapse.

\section{BILLETER Jean-François, 2001}

L'art chinois de l'écriture : essai sur la calligraphie, Genève/Paris, Skira.

\section{CASSIRER Ernst, 1995}

«Qu'est-ce que la beauté ? » in Écrits sur l'art, CAPEILLIÈRES Fabien (dir., trad.), Paris, Éd. du Cerf, pp. 123-127.

\section{CHARRIER Isabelle, 1991}

La peinture japonaise contemporaine de 1750 à nos jours, Besançon, La Manufacture.

\section{CHARRIER Isabelle, 1999}

«La réaction nationaliste dans les milieux artistiques », in TSCHUDIN Jean-Jacques \& HAMON Claude (dir.), La nation en marche. Études sur le Japon impérial de Meiji, Arles, Éd. Philippe Picquier, pp. 163-180.

\section{CHISOLM Lawrence, 1963}

Fenollosa: The Far East and American Culture, New Haven, Yale University Press.

\section{CONANT Ellen P. (ed.), 1995}

Nihonga, Transcending the Past: Japanese-Style Painting, 1868-1968, St. Louis (Mo), St. Louis Art Museum.

CONANT Ellen P., 2006 "Japan "Abroad" at the Chicago
Exposition, 1893 ", in CONANT Ellen P. (ed.), Challenging Past and Present: The Metamorphosis of $19^{\text {th }}$ Century Japanese Art, Honolulu, University of Hawaii Press, pp. 254-280.

\section{CROISSANT Doris, 2006}

«In Quest of the Real: Portrayal and Photography in Japanese Painting Theory ", in CONANT Ellen P. (ed.), Challenging Past and Present: The Metamorphosis of 19th Century Japanese Art, Honolulu, University of Hawaii Press, pp. 153-176.

\section{DERRIDA Jacques, [1967] 1997}

De la Grammatologie, Paris, Éd. de Minuit.

FENOLLOSA Ernest, [1912] 1963 Epochs of Chinese and Japanese Art, 2 vol., New York, Dover Publications [éd. or. : Londres/New York, Heinemann].

FENOLLOSA Ernest, [1912] 1913 L'art en Chine et au Japon, trad. Gaston Migeon, Paris, Hachette [éd. or. : Epochs of Chinese and Japanese Art, Londres/ New York, Heinemann].

\section{FENOLLOSA Ernest, 1916}

"Noh" or Accomplishment: A Study of the Classical Stage of Japan, London, Macmillan and Co.

\section{FENOLLOSA Ernest, [1882] 1989}

Bijutsu shinsetsu 美術真説 (Discours sur la vraie théorie des arts), 
in AOKI Shigeru 青木茂 \& SAKAI

Tadayasu 酒井忠康 (dir.), Nihon kindai

shisō taikei 日本近代思想大系 (Collection

d'œuvres de la pensée moderne),

Tokyo, Iwanami shoten 岩波書店, vol. 17, pp. 35-65.

\section{FENOLLOSA Ernest, 2009}

Ernest Francisco Fenollosa: Published

Writings in English, édité par Yamaguchi

Seiichi, 3 vol., Tokyo, Synapse.

\section{FENOLLOSA Ernest}

\& POUND Ezra, 1972

Le Caractère écrit chinois, matériau

poétique, trad. Ghislain Sartoris, Paris,

L'Herne.

\section{FLAHAULT François, 2003}

«Théorie de la culture et catégories esthétiques », in ESCANDE Yolaine \& SCHAEFFER Jean-Marie (dir.), L'esthétique : Europe, Chine et ailleurs, Paris, Éd. You-Feng, pp. 127 -137.

\section{FREEDMAN Jonathan, 1990}

Professions of Taste, Henry James, British Aestheticism and Commodity Culture, Stanford (Cal.), Stanford University Press.

\section{FUKUCHI Mataichi}

\& KINO Toshio (dir.), [1900] 2005

Histoire de l'art du Japon [1900, ouvrage de la Commission impériale pour I'Exposition universelle de Paris], Tokyo, Synapse.

\section{GENETTE Gérard, 1997}

L'Éuvre de l'art, t. II : La Relation esthétique, Paris, Seuil.
GONSE Louis, [1883] 2003

L'Art japonais, Tokyo/Londres,

Synapse/Ganeshal.

GUTH Christine, 2006

"Meiji Response to Bunjinga », in

CONANT Ellen P. (ed.), Challenging

Past and Present: The Metamorphosis

of $19^{\text {th }}$ Century Japanese Art, Honolulu, University of Hawaii Press, pp. 177-193.

\section{HAMERTON Philip G., 1864a}

"Analysis and Synthesis in Painting ",

Fine Arts Quarterly Review,

mai, 2 : 236-254.

\section{HAMERTON Philip G., 1864b}

"The Reaction from Pre-Raphaelitism », Fine Arts Quarterly Review, mai, 2 : 254-262.

\section{HEGEL Georg W.F., 1997}

Esthétique, traduction de Charles Bénard, Benoît Timmermans et Paolo Zaccaria, Paris, Librairie générale française, coll. « Livre de Poche », 2 vol.

INAGA Shigemi, 2001a

«Claude Monet Between "Impressionism" and "Japonism" ", in SPATE Virginia et al ., Monet and Japan, Sydney, National Gallery of Australia, pp. 64-76.

\section{INAGA Shigemi, 2001b}

«Un destin de pensée : l'impact d'Okakura Kakuzō (Tenshin) sur le développement de l'histoire de l'art en Inde et au Japon au début du xx siècle ", in MONNET Livia (dir.), Approches 
critiques de la pensée japonaise du $x x^{e}$ siècle, Montréal, Presses universitaires de Montréal, pp. 329-348.

INAGA Shigemi, 2004-2005

«Images changeantes de l'art japonais: depuis la vue impressionniste du Japon à la controverse de l'esthétique orientale (1860-1940) ", Journal of the Faculty of Letters, University of Tokyo, 29-30 : 73-86.

INAGA Shigemi, 2011

«Philosophy, Ethics and Aesthetics in the Far-Eastern Cultural Sphere », in INAGA Shigemi (ed.), Questioning Oriental Aesthetics and Thinking: Conflicting Visions of "Asia" under the Colonial Empires, Actes du $38^{\mathrm{e}}$ colloque international de I'International Research Center for Japanese Studies, Kyoto, Kokusai Nihon bunka kenkyū sentā 国際 日本文化研究センター, pp. 31-45.

\section{KARATANI Kōjin 柄谷行人, 2004}

«Bijutsukan to shite no rekishi : Okakura Tenshin to Fenorosa » 美術館としての歴史 —— 岡倉天心とフェノロサ (De l'histoire considérée comme un musée : Okakura Tenshin et Fenollosa), Teihon Karatani Köjin shū 4. Nēshon to bigaku 定本柄谷行人集 4 ネーションと美学 (CFuvres de Karatani Kōjin, t. IV : Nation et esthétique), Tokyo, Iwanami shoten 岩波書店, pp. 127-147. Version anglaise (originale), 1994 : "Japan as a Museum », in MUNROE Alexandra, Japan Art after 1946: Scream against the Sky, New York, H. N. Abrams, pp. 33-39.
KINOSHITA Nagahiro 木下長宏, 2005 Okakura Tenshin : mono ni kan zureba tsui ni ware nashi 岡倉天心 —— 物二 観ズレバ竟二吾無シ (Okakura Tenshin. «Contempler les choses conduit à perdre son moi »), Kyoto, Mineruva shobō ミネルヴァ書房.

KURIHARA Shin.ichi 栗原信一, 1963

Fenorosa to Meiji bunka フェノロサと明治 文化 (Fenollosa et la culture de l'ère Meiji), Tokyo, Rikugei shobō 六芸書房.

\section{KUROKAWA Mayori 黒川真頼，} [1878] 1888

Kōgei shiryō 工藝志料 (Histoire des arts manuels du Japon), Tokyo, Yūrindō 有隣堂.

LIPPIT Yukio \& MURAI Noriko, 2012 Beyond Tenshin: Okakura Kakuzō's Multiple Legacies, Jōsai University Review of Japanese Culture and Society, décembre, 24.

\section{LUCKEN Michael, 2001}

L'Art du Japon au xx siècle : pensée, forme, résistances, Paris, Hermann.

\section{MARQUET Christophe, 1995}

Le peintre Asai Chū (1856-1907) et le monde des arts à l'époque Meiji, thèse de doctorat sous la direction de Jean-Jacques Origas, INALCO.

\section{MARQUET Christophe, 1999}

«Conscience patrimoniale et écriture de I'histoire de l'art national », in TSCHUDIN Jean-Jacques \& HAMON Claude (dir.), La nation en marche. Études sur le Japon 
impérial de Meiji, Arles, éd. Philippe

Picquier, pp. 143-162.

\section{MARQUET Christophe, 2002}

« Le Japon moderne face à son

patrimoine artistique », Cipango, hors-

série «Mutations de la conscience dans

le Japon moderne » : 243-304.

\section{MARQUET Christophe, 2005}

«Défense et illustration de l'art national à la fin du xIx siècle : la création de la revue Kokka ", Revue d'études japonaises du CEEJA. Benkyōkai 勉強会, Aurillac, Presses orientalistes de France, $1: 289-314$.

\section{MARQUET Christophe, 2008}

« Jūkyū seiki kōhan no Furansu ni okeru Nihon bijutsu shigaku no reimeiki :

L'Art japonais oyobi Ukiyo-e ruikō to Histoire de l'art du Japon »

十九世紀後半のフランスにおける

日本美術史学の黎明期——L'Art japonais』及び『浮世絵類考』と

『Histoire de l'art du Japon』, «The Dawn of Japanese Art History

in France During the Second Half

of the Nineteenth Century:

L'Art japonais, Ukiyo-e ruikō and

Histoire de l'art du Japon ",

Hikaku nihongaku kenkyū sentā

kenkyū nenpō 比較日本学研究センター 研究年報 Center for Comparative Japanese Studies. Annual Bulletin, vol. 4, Tokyo, université Ochanomizu, mars, pp. 71-97.

\section{MARQUET Christophe}

\section{\& BUTEL Jean-Michel (dir.), 2009}

L'invention des « arts populaires»: Yanagi
Sōetsu et le Mingei, numéro spécial, Cipango. Cahiers d'études japonaises, 16.

MICHAUD Yves, 2003

«Évaluations et prescriptions. En général et aujourd'hui en particulier », Revue francophone d'Esthétique, Aix-enProvence, 1 : 47-57.

MORI Tōgo 森東吾, 1987

« Fenorosa ni okeru Supensā to Hēgeru » フェノロサにおけるスペンサーとヘーゲル

(Spencer et Hegel chez Fenollosa), Lotus. Nihon Fenorosa gakkai kikanshi Lotus 日本フェノロサ学会機関誌, mars, 7 : 14-30.

\section{MURAKATA Akiko 村形明子, 1983} « Bijutsu shinsetsu to Fenorosa ikō » 『美術真説』とフェノロサ遺稿 («Le Bijutsu shinsetsu et le manuscrit de Fenollosa de $1881 »)$, Eibungaku hyōron 英文学評論 (Bulletin de la Société de littérature anglaise), université de Kyoto, 49 : 45-6. Source en ligne : http://repository. kulib.kyoto-u.ac.jp/dspace/bitstream/ 2433/135170/1/ebk00049_045b.pdf

MURAKATA Akiko, 2000-2001

Ānesuto F. Fenorosa bunsho shūsei : honkoku, hon.yaku to kenkyū アーネスト・F・フェノロサ文書集成— 翻刻、翻訳と研究 (Bibliographie des œuvres d'Ernest F. Fenollosa : réédition, traduction, étude critique), 2 vol., Kyoto, Kyoto daigaku gakujutsu shuppankai 京都大学学術出版会. 
OKAKURA Kakuzō 岡倉覚三（角蔵）, [1903] 1917

Les Idéaux de l'Orient ; suivi du Réveil du Japon, trad. Jenny Serruys, Paris, Payot [éd. or. : The Ideals of the East: With Special Reference to the Art of Japan, Londres, J. Murray].

\section{OKAKURA Kakuzō, 1979-1981}

Okakura Tenshin zenshū 岡倉天心全集

(Fuvres complètes d'Okakura Tenshin), 8 vol., Tokyo, Heibonsha 平凡社.

\section{OKAKURA Kakuzō, 1984}

Collected English Writings, 3 vol., Tokyo, Heibonsha.

\section{OKAKURA Kakuzō, [1906] 2000}

Le Livre du thé, trad. Gabriel Mourey, Paris, Dervy [éd. or. : The Book of Tea, New York, Duffield].

\section{OKAKURA Kakuzō, 2001a}

Nihon bijutsu shi 日本美術史 (Histoire de l'art japonais), Tokyo, Heibonsha 平凡社.

\section{OKAKURA Kakuzō, 2001b}

《Taitō kōgei shi » 泰東巧藝史 (Histoire de la création artistique en Asie orientale), in Nihon bijutsu shi 日本 美術史 (Histoire de l'art japonais), Tokyo, Heibonsha 平凡社, pp. 264-348.

\section{«Okakura Tenshin. Geijutsu kyōiku} no ayumi » ten jikkō iinkai (ed.)

「岡倉天心芸術教育の步み」展実行委員会

(Commission de l'exposition «Okakura Tenshin : le chemin de l'éducation artistique »), 2010 Ima Tenshin o kataru. Tōkyō geijutsu daigaku sōritsu hyakunijū shūnen

Okakura Tenshin ten kinen shinpojiumu

いま天心を語る 一一東京藝術大学創立 120周年岡倉天心展記念シンポジウム (Raconter Okakura Tenshin aujourd'hui. Actes du colloque Okakura Tenshin célébrant les 120 ans de la fondation de l'université des beaux-arts de Tokyo), Tokyo, Tokyo geijutsu daigaku shuppankai 東京藝術大学出版会.

Okakura Tenshin kenkyūkai (ed.) 岡倉天心研究会編, 2005 Watariumu bijutsukan no Okakura Tenshin kenkyūkai ワタリウム美術館の岡倉 天心·研究会 (Actes du Colloque Okakura Tenshin organisé par le musée d'art contemporain Watarium), Tokyo, Yūbun shoin 右文書院.

\section{PRETTEJOHN Elizabeth, 2007}

Art for Art's Sake. Aestheticism in Victorian Painting, New Haven, Yale University Press.

\section{RABATÉ Dominique, 2007}

"La valeur comme question », Modernités, numéro L'art et la question de la valeur, Bordeaux, Presses universitaires de Bordeaux, 25 : 9-25.

RIMER J. Thomas, 2001 " Hegel in Tokyo », in MARRA Michael F., A History of Modern Japanese Aesthetics, Honolulu, University of Hawaii Press, pp. 97-107.

\section{SCHAEFFER Jean-Marie, 2003}

«Relativité culturelle ou universalité anthropologique ? Faux débats et vraies questions », in ESCANDE Yolaine 
\& SCHAEFFER Jean-Marie (dir.), L'esthétique: Europe, Chine et ailleurs, Paris, éd. You-Feng, pp. 139-151.

SHIMIZU Emiko 清水恵美子, 2012 Okakura Tenshin no hikaku bunkashiteki kenkyū 岡倉天心の比較文化史的研究 (Okakura Tenshin du point de vue de I'histoire culturelle comparative), Kyoto, Shibunkaku shuppan 思文閣出版.

TAKASHINA Shūji 高階秀爾,

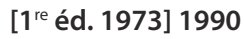

Nihon kindai bijutsushi-ron 日本近代 美術史論 (Essais sur l'histoire de l'art du Japon moderne), Tokyo, Kōdansha gakujutsu bunko 講談社学術文庫.

TAKASHINA Shūji, 1993

Nihon kindai no bi ishiki 日本近代の美意識 (La conscience esthétique dans le Japon moderne), Tokyo, Seidosha 青土社.

TAKI Teizō 瀧悌三, 1993

Nihon kindai bijutsu jikenshi 日本近代 美術事件史 (Histoire événementielle de l'art moderne japonais), Osaka, Tōhō shuppan 東方出版.

TSENG Alice, 2008

The Imperials Museums of Meiji Japan, Washington, University of Washington Press.

\section{WESTON Victoria, 2004}

Japanese Painting and National Identity: Okakura Tenshin and his Circle, Ann Arbor, Center for Japanese Studies, The University of Michigan.
YAMAGUCHI Seiichi 山口静一, 1984

Fenorosa : Nihon bunka no sen.yō ni sasageta isshō フェノロサー日本文化の 宣揚に捧げた一生 (Fenollosa : une vie entière consacrée à la promotion de la culture japonaise), 2 vol., Tokyo, Sanseidō 三省堂.

YAMAGUCHI Seiichi, 1993

«Fenorosa to bunjinga » フェノロサと文人画 (Fenollosa et la peinture de lettrés), Lotus. Nihon Fenorosa gakkai kikanshi Lotus 日本フェノロサ学会機関誌, mars, 13 : 1-19. 